\title{
ON OPTIMAL TAXES AND SUBSIDIES: A DISCRETE SADDLE-POINT THEOREM WITH APPLICATION TO JOB MATCHING UNDER CONSTRAINTS
}

\author{
Koji Yokote \\ Waseda University, Japan \\ sidehandetoki.waseda.jp
}

\begin{abstract}
When a government intervenes in markets by setting a target amount of goods/services traded, its tax/subsidy policy is optimal if it entices the market participants to obey the policy target while achieving the highest possible social welfare. For the model of job market interventions by Kojima et al. (2019), we establish the existence of optimal taxes/subsidies as well as their characterization. Our methodological contribution is to introduce a discrete version of Karush-Kuhn-Tucker's saddle-point theorem based on the techniques in discrete convex analysis. We have two main results: we (i) characterize the optimal taxes/subsidies and the corresponding equilibrium salaries as the minimizers of a Lagrange function, and (ii) prove that the function satisfies a notion of discrete convexity (called $\mathrm{L}^{\natural}$-convexity). These results together with others imply that an optimal tax/subsidy level exists and can be calculated via a computationally efficient algorithm.
\end{abstract}

Keywords: Job matching, taxation, discrete convex analysis.

JEL Classification Numbers: C61, C78, D47, H21.

The author is grateful to the editor, two anonymous referees, and seminar participants at Osaka University of Economics and Tokyo Metropolitan University, for their valuable comments. This work was supported by JSPS KAKENHI Grant Numbers 17H07179, 20K13458, and Waseda University Grants for Special Research Projects Grant Number 2019C-015. 


\section{INTRODUCTION}

overnments often intervene in markets in order to address socio-economic $\checkmark$ problems such as environmental pollution or inequality. A representative form of government interventions is that the government (or international organizations) first determines a target amount of goods/services traded and then introduces taxes/subsidies accordingly. ${ }^{1}$ Three examples are as follows:

- Some countries/cities set a policy goal regarding the usage of fossil fuel-powered cars and electric cars in an attempt to decrease the number of the former and increase the number of the latter (see Burch and Gilchrist 2018 for a survey). Taxes/subsidies are introduced to achieve the goal.

- In 2019, the G20 countries reached an agreement to reduce additional marine plastic waste to zero by $2050 .^{2}$ Imposing consumption taxes on plastic products is a hot environmental issue lately. ${ }^{3}$

- In the job market, the government often introduces taxes/subsidies to firms in an attempt to achieve a policy goal concerning diversity and/or equality. ${ }^{4}$

In these situations, an ideal tax/subsidy policy would entice the market participants to obey the policy target while achieving the highest possible social welfare; such a policy is called optimal hereafter. The purpose of this paper is to take a discrete mathematics approach to optimal taxes/subsidies and address theoretical issues of existence, characterization, and efficient computation. We consider the model of job market interventions by Kojima et al. (2019), which is suitable for analyzing the third example mentioned above.

1 The basic idea of this tax scheme can be traced back to Baumol and Oates (1971). Note the difference from the well-known Pigovian taxes determined by the level of externalities. For an application of the latter tax scheme, see Parry et al. (2007).

2 The Japan Times, 2020-7-16 (accessed on 2020-9-7).

3 In Chicago, a 7-cent tax on all grocery bags led to a 42 percent drop in usage; see Newsday, 2019-8-11 (accessed on 2020-9-7).

4 The Japanese government provides a subsidy to a firm if $2.2 \%$ of its workforce consists of people with disabilities; see Japanese Ministry of Health, Labour and Welfare (in Japanese, accessed on 2020-9-7). Other related examples in job markets can be found in Kojima et al. (2019). 
Our analysis depends on admittedly restrictive assumptions; we assume that (i) all the participants have a quasi-linear utility function (i.e., no income effect is allowed), ${ }^{5}$ (ii) all the firms view workers as substitutes (i.e., no complementarity is allowed), and (iii) the participants' valuations/revenues are known to the social planner. However, we believe that our analysis serves as a preliminary step for solving more complicated problems. To facilitate applications in future research, we provide a detailed discussion of the underlying mathematical tool.

In the first part of the paper, we introduce a preliminary mathematical tool. We borrow techniques from discrete convex analysis (Murota 2003a) and introduce a discrete version of Karush-Kuhn-Tucker's theorem, termed the discrete saddle-point theorem. ${ }^{6}$ This theorem concerns a constrained maximization problem of a function defined over a discrete domain. Its major advantage is that it enables a unified treatment of constraints and discrete variables: the former is used to capture the government's policy goal and the latter is used to deal with indivisible commodities and discuss computational problems.

In the second part, we apply the discrete saddle-point theorem to the model of job market interventions developed by Kojima et al. (2019). ${ }^{7}$ In this model, workers and firms participate in bilateral contracts associated with wages and the government sets the maximum/minimum number of workers allowed to be hired by each firm. ${ }^{8}$ Applying the discrete saddle-point theorem to a suitably defined maximization problem, we (i) characterize the optimal taxes/subsidies and the corresponding equilibrium salaries as the minimizers of a Lagrange function, and (ii) prove that the function satisfies a notion of discrete convexity (called $L^{\natural}$-convexity). These results together with Kojima et al.'s (2019) result imply that an optimal tax/subsidy level exists and can be calculated via a computationally efficient algorithm.

This paper is part of the literature on the application of discrete convex

5 Quasi-linearity means that utilities are linear with respect to money. We note that Kojima et al. (2019) do not assume quasi-linearity for doctors.

6 Mathematically, this theorem is an immediate corollary of the well-known intersection theorem in discrete convex analysis but is tailored to the use of economists by emphasizing its parallelism with the original theorem.

7 This model is a generalization of that of Kelso and Crawford (1982).

8 These constraints are called "interval constraints." Precisely, Kojima et al. (2019) (see also Kojima et al. (2020a)) consider a general form of constraints and prove that the substitutes condition is preserved if and only if the constraints are "generalized interval constraints," a slight generalization of interval constraints. 
analysis in economics; see Murota (2016) for a survey. Examples or prior applications include matching models (Kojima et al. 2018) and trading networks (Candogan et al. 2016). To the best of our knowledge, this paper is the first to apply discrete convex analysis to the analysis of taxes/subsidies.

This paper also contributes to the literature on matching with constraints. One can find different theoretical approaches from ours, as well as various forms of constraints in real-world problems, in Kamada and Kojima (2017) or Kamada and Kojima (2020).

Dupuy et al. (2020) develop a model of matching with taxation that accommodates a general form of taxes beyond linear ones and conduct a welfare analysis from both theoretical and econometric perspectives. Our approach is distinguished from theirs in that constraints on hiring and the computation of taxes are discussed. The extensive literature on taxation can be found in the reference therein.

The remainder of this paper is organized as follows. Section 2 introduces basic concepts in discrete convex analysis and presents the discrete saddle-point theorem. Section 3 analyzes optimal taxes/subsidies in a job-matching model. Section 4 presents concluding remarks. All proofs are provided in Section 5.

\section{MATHEMATICAL PRELIMINARY: A DISCRETE SADDLE-POINT THEOREM}

In the handling of mathematical problems with constraints, Karush-KuhnTucker's saddle-point theorem plays a central role. In this section, we introduce a discrete version of the theorem by utilizing the concepts in discrete convex analysis (Murota 2003a).

Let $K$ be a finite set. Let $\mathbb{R}^{K}$ denote the real vector space indexed by the elements in $K$. Let $\mathbb{Z}^{K} \subseteq \mathbb{R}^{K}$ be the set of vectors with integer coordinates. For a function $f: \mathbb{Z}^{K} \rightarrow \mathbb{Z} \cup\{-\infty\}$, we define the effective domain of $\boldsymbol{f}(\cdot)$ by

$$
\operatorname{dom} f=\left\{x \in \mathbb{Z}^{K}: f(x)>-\infty\right\} .
$$

For $A \subseteq K$, let $\mathbb{1}^{A} \in\{0,1\}^{K}$ denote the characteristic vector of $\boldsymbol{A}$, i.e.,

$$
\mathbb{1}_{k}^{A}= \begin{cases}1 & \text { if } k \in A \\ 0 & \text { otherwise }\end{cases}
$$

For a singleton set $\{k\} \subseteq K$, we write $\mathbb{1}^{k}$ for $\mathbb{1}^{\{k\}}$. 
For $x \in \mathbb{Z}^{K}$, we define

$$
\operatorname{supp}^{+} x=\left\{k \in K: x_{k}>0\right\}, \operatorname{supp}^{-} x=\left\{k \in K: x_{k}<0\right\} .
$$

A function $f: \mathbb{Z}^{K} \rightarrow \mathbb{Z} \cup\{-\infty\}$ with $\operatorname{dom} f \neq \emptyset$ is said to be $\mathbf{M}^{\natural}$-concave (Murota 2003a) if for any $x, y \in \mathbb{Z}^{K}$ and $k \in \operatorname{supp}^{+}(x-y)$, we have

(i) $f(x)+f(y) \leq f\left(x-\mathbb{1}^{k}\right)+f\left(y+\mathbb{1}^{k}\right)$, or

(ii) there exists $\ell \in \operatorname{supp}^{-}(x-y)$ such that $f(x)+f(y) \leq f\left(x-\mathbb{1}^{k}+\mathbb{1}^{\ell}\right)+$ $f\left(y+\mathbb{1}^{k}-\mathbb{1}^{\ell}\right)$.

Intuitively, this condition states that if two points $x$ and $y$ approach each other, then the sum of the function values weakly increases. For a discussion of this property, see Chapter 6 of Murota (2003a) or Section 3 of Kojima et al. (2018). The class of $\mathrm{M}^{\natural}$-concave functions includes various standard functions such as linear functions and quadratic functions; see Section 6.3 of Murota (2003a) for other examples.

For $x, y \in \mathbb{Z}^{K}$, we define $x \wedge y \in \mathbb{Z}^{K}$ and $x \vee y \in \mathbb{Z}^{K}$ by $(x \wedge y)_{k}=\min \left\{x_{k}, y_{k}\right\}$ for all $k \in K,(x \vee y)_{k}=\max \left\{x_{k}, y_{k}\right\}$ for all $k \in K$. A function $f: \mathbb{Z}^{K} \rightarrow \mathbb{Z} \cup\{-\infty\}$ with $\operatorname{dom} f \neq \emptyset$ is said to be $\mathbf{L}^{\natural}$-concave (Murota 2003a) if for any $x, y \in \operatorname{dom} f$ and $^{9} \lambda \in \mathbb{Z}_{+}$, we have

$$
f(x)+f(y) \leq f\left(\left(x+\lambda \cdot \mathbb{1}^{K}\right) \wedge y\right)+f\left(x \vee\left(y-\lambda \cdot \mathbb{1}^{K}\right)\right) .
$$

$\mathrm{M}^{\natural}$-concavity and $\mathrm{L}^{\natural}$-concavity are connected via the conjugacy relationship; see Theorem 8.12 of Murota (2003a). A function $f(\cdot)$ is said to be $\mathbf{L}^{\natural}$-convex if $-f(\cdot)$ is $\mathrm{L}^{\natural}$-concave. $\mathrm{M}^{\natural}$-convexity is defined analogously.

We now turn our attention to constrained maximization problems with discrete variables. Recall that Karush-Kuhn-Tucker's saddle-point theorem holds if the objective and constraint functions are concave. With this in mind, we can consider replacing "concavity" with " $\mathrm{M}^{\natural}$-concavity." This idea is stated below, where all the functions are assumed to be defined on $\mathbb{Z}^{K}$.

Naive idea. Consider the problem of maximizing an objective function subject to constraint functions. If the objective and constraint functions satisfy $M^{\natural}$ concavity, then the solutions to the problem are translated into the saddle points of a Lagrange function.

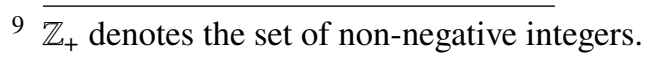


Unfortunately, this idea does not work, even if the constraint functions are linear; we provide a counterexample in Section S.1 of the Supplemental Material (Yokote 2020). To circumvent this difficulty, we restrict the class of constraint functions to linear functions with a certain discrete structure. We say that $\mathcal{K} \subseteq 2^{K} \backslash\{\emptyset\}$ is a hierarchy ${ }^{10}$ if, for every pair of elements $A$ and $A^{\prime}$ in $\mathcal{K}$, we have $A \subseteq A^{\prime}$ or $A^{\prime} \subseteq A$ or $A \cap A^{\prime}=\emptyset$. We say that a set of functions $g_{1}, \ldots, g_{q}: \mathbb{Z}^{K} \rightarrow \mathbb{Z}$ is a hierarchical set of affine functions if

(i) for each $j=1, \ldots, q$, there exist $a_{j} \in \mathbb{Z}$ and $A_{j} \subseteq K$ with $A_{j} \neq \emptyset$ such that $g_{j}(x)=a_{j}-x \cdot \mathbb{1}^{A_{j}}$ for all $x \in \mathbb{Z}^{K}$, and

(ii) the set $\left\{A_{j}: j=1, \ldots, q\right\}$ is a hierarchy.

We are in a position to state our first theorem.

Theorem 1 (Discrete Saddle-Point Theorem). Let $f: \mathbb{Z}^{K} \rightarrow \mathbb{Z} \cup\{-\infty\}$ be an $M^{\natural}$-concave function and $g_{1}, \ldots, g_{q}: \mathbb{Z}^{K} \rightarrow \mathbb{Z}$ be a hierarchical set of affine functions. Then, for $x^{*} \in \mathbb{Z}_{+}^{K}$, the following are equivalent:

(i) $x^{*}$ is a solution to $\max f(x)$ subject to $g_{j}(x) \geq 0$ for all $j=1, \ldots, q$.

(ii) There exists $\left(\lambda_{1}^{*}, \ldots, \lambda_{q}^{*}\right) \in \mathbb{Z}_{+}^{q}$ such that

$$
L\left(x, \lambda^{*}\right) \leq L\left(x^{*}, \lambda^{*}\right) \leq L\left(x^{*}, \lambda\right) \text { for all } x \in \mathbb{Z}^{K}, \lambda \in \mathbb{Z}_{+}^{q},
$$

where $L(\cdot, \cdot): \mathbb{Z}^{K} \times \mathbb{Z}_{+}^{q} \rightarrow \mathbb{Z} \cup\{-\infty\}$ is given by

$$
L(x, \lambda)=f(x)+\sum_{j=1}^{q} \lambda_{j} g_{j}(x) \text { for all } x \in \mathbb{Z}^{K}, \lambda \in \mathbb{Z}_{+}^{q} .
$$

Moreover, $\left(\lambda_{1}^{*}, \ldots, \lambda_{q}^{*}\right)$ satisfies

$$
\lambda_{j}^{*} \cdot g_{j}\left(x^{*}\right)=0 \text { for all } j=1, \ldots, q \text {. }
$$

Proof. See Section 5.1. ${ }^{11}$

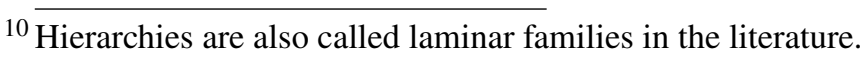

${ }^{11}$ We remark that (3) is not employed in showing (ii) $\Rightarrow$ (i). 
In the literature, (3) is called the complementary slackness.

As shown in the proof, this theorem is an immediate corollary of the well-known intersection theorem in discrete convex analysis (Theorem 8.17 of Murota (2003a)). However, it presents a familiar form for economists, as it is similar to Karush-Kuhn-Tucker's theorem.

Remark 1. Section 8.4 of Murota (2003a) and Section 6 of Murota (1998) develop a general Lagrange duality theory for an integer problem. As demonstrated there, under the M-convexity assumption, strong duality holds, and the solutions to the primal/dual problems can be translated into the saddle point of a Lagrange function. The present approach is distinguished from this result in that we explicitly refer to constraint functions and formulate the Lagrange function incorporating them.

\section{OPTIMAL TAXES/SUBSIDIES IN A JOB-MATCHING MODEL}

This section applies the mathematical tool in Section 2 to a market model. We consider the model of job-market interventions due to Kojima et al. (2019), which accommodates hospital-intern markets as a representative example. While the authors do not assume quasi-linearity for doctors, we assume it for analytical purposes.

\subsection{Underlying Framework}

Let $D$ denote the set of doctors and $H$ the set of hospitals. A matching is represented by a function $\mu: D \rightarrow \bar{H}$, where $\bar{H} \equiv H \cup\left\{h_{0}\right\}$, and $h_{0}$ stands for an outside option; with a slight abuse of notation, we write $\mu(h) \equiv\{d \in D$ : $\mu(d)=h\}$ for $h \in H$. Let $\Omega \equiv D \times H$. A salary system is a vector $s \in \mathbb{R}^{\Omega}$.

Each doctor $d$ has a valuation function $v_{d}: \bar{H} \rightarrow \mathbb{Z}$. For $s \in \mathbb{R}^{\Omega}$, d's utility function is given by

$$
v_{d}[s](h)=v_{d}(h)+s_{(d, h)} \text { for all } h \in \bar{H},
$$

where $s_{\left(d, h_{0}\right)}$ is defined to be 0 . We define $d$ 's indirect utility function $V_{d}: \mathbb{R}^{\Omega} \rightarrow \mathbb{R}$ by

$$
V_{d}(s)=\max _{h \in \bar{H}} v_{d}[s](h) \text { for all } s \in \mathbb{R}^{\Omega} .
$$


Each hospital $h$ has a revenue function $v_{h}: 2^{D} \rightarrow \mathbb{Z}$. We define $h^{\prime} s$ demand correspondence $X_{h}: \mathbb{R}^{\Omega} \rightarrow 2^{D}$ by

$$
\begin{array}{r}
X_{h}(s)=\left\{A \subseteq D: v_{h}(A)-\sum_{d \in A} s_{(d, h)} \geq v_{h}\left(A^{\prime}\right)-\sum_{d \in A^{\prime}} s_{(d, h)} \text { for all } A^{\prime} \subseteq D\right\} \\
\text { for all } s \in \mathbb{R}^{\Omega} .
\end{array}
$$

We make the following assumptions on $v_{h}(\cdot)$, where the latter one is due to Kelso and Crawford (1982):

- Monotonicity: for any $A, A^{\prime} \subseteq D$ with $A \subseteq A^{\prime}$, we have $v_{h}(A) \leq$ $v_{h}\left(A^{\prime}\right)$.

- Gross substitutes condition: for any $s, s^{\prime} \in \mathbb{R}^{\Omega}$ with $s \leq s^{\prime}$ and $A \in$ $X_{h}(s)$, there exists $A^{\prime} \in X_{h}\left(s^{\prime}\right)$ such that $\left\{d \in A: s_{(d, h)}=s_{(d, h)}^{\prime}\right\} \subseteq A^{\prime}$.

Proposition 1 (Fujishige and Yang 2003). $v_{h}(\cdot)$ satisfies the gross substitutes condition if and only if $v_{h}(\cdot)$ is $M^{\natural}$-concave. ${ }^{12}$

In real job-matching markets, the government often imposes constraints on hospitals' hiring. Kojima et al. (2019) note that "[r]estrictions on the number of hires are commonplace, and these naturally exist in the form of interval constraints." An interval constraint consists of a floor constraint (minimum number of workers hired) and a ceiling constraint (maximum number of workers hired). Formally, for each $h \in H$, let $\underline{\delta}_{h}, \bar{\delta}_{h} \in\{0,1, \ldots,|D|\}$ with $\underline{\delta}_{h} \leq \bar{\delta}_{h}$ denote the floor constraint and the ceiling constraint, respectively. We define $h$ 's feasibility collection $\mathcal{F}_{h}$ by

$$
\mathcal{F}_{h}=\left\{A \subseteq D: \underline{\delta}_{h} \leq|A| \leq \bar{\delta}_{h}\right\}
$$

A transfer policy $t=\left(t_{h}\right)_{h \in H} \in \mathbb{Z}^{H}$ specifies the government transfer to each hospital $h$ as $t_{h}$ times the number of its hires; $t_{h}>0$ indicates a subsidy, and $t_{h}<0$ represents a tax. For $s \in \mathbb{R}^{\Omega}$ and $t \in \mathbb{R}^{H}, h$ 's post-transfer profit

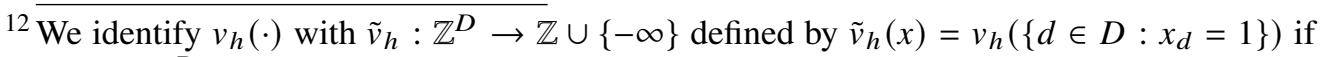
$x \in\{0,1\}^{D}$ and $\tilde{v}_{h}(x)=-\infty$ otherwise. 
function is given by ${ }^{13}$

$$
v_{h}[s, t](A)=v_{h}(A)+|A| t_{h}-\sum_{d \in A} s_{(d, h)} \text { for all } A \subseteq D,
$$

where the summation over the empty set is defined to be 0 . We define $h$ 's profit function $V_{h}: \mathbb{R}^{\Omega} \times \mathbb{R}^{H} \rightarrow \mathbb{R}$ by

$$
V_{h}(s, t)=\max _{A \subseteq D} v_{h}[s, t](A) \text { for all }(s, t) \in \mathbb{R}^{\Omega} \times \mathbb{R}^{H} .
$$

\subsection{Equilibrium, Desirable Matching, and Optimal Transfer Policy}

The government aims to realize a desirable matching in a market equilibrium, with desirability being tested in terms of feasibility and efficiency. We introduce additional concepts to discuss this goal formally.

A triple $(\mu, s, t)$ forms an uncompelled competitive equilibrium if

(i) for any $d \in D, v_{d}[s](\mu(d)) \geq v_{d}[s](h)$ for all $h \in \bar{H}$, and

(ii) for any $h \in H, v_{h}[s, t](\mu(h)) \geq v_{h}[s, t](A)$ for all $A \subseteq D$.

Kojima et al. (2019) introduce this equilibrium concept and prove its existence. Importantly, the hospitals are not compelled to obey the interval constraints in this definition. We call $s$ an equilibrium salary.

We say that a matching $\mu$ is feasible if $\mu(h) \in \mathcal{F}_{h}$ for all $h \in H$. For a matching $\mu$, we define the sum of valuations/revenues by

$$
\mathbf{V}(\mu)=\sum_{d \in D} v_{d}(\mu(d))+\sum_{h \in H} v_{h}(\mu(h))
$$

We say that $\mu$ is constrained efficient if $\mu$ is feasible and, for any feasible matching $\mu^{\prime}$, it holds that $\mathbf{V}(\mu) \geq \mathbf{V}\left(\mu^{\prime}\right)$. The following lemma provides a sufficient condition for a matching in an equilibrium to be constrained efficient:

${ }^{13}$ If $v_{h}(\cdot)$ satisfies the gross substitutes condition, then $v_{h}[s, t](\cdot)$ also satisfies the condition. This claim can be verified by appeal to basic operations of $\mathrm{M}^{\natural}$-concave functions; see Theorem 6.15 of Murota (2003a). Kojima et al. (2020b) consider a general form of transfer policies and provide a necessary and sufficient condition under which a policy preserves the substitutes condition of the revenue function. 
Lemma 1. Let $(\mu, s, t)$ be an uncompelled competitive equilibrium such that $\mu$ is feasible. If the pair $(\mu, t)$ satisfies

$$
\left[t_{h}>0 \Longrightarrow|\mu(h)|=\underline{\delta}_{h}\right] \text { and }\left[t_{h}<0 \Longrightarrow|\mu(h)|=\bar{\delta}_{h}\right]
$$

then $\mu$ is constrained efficient.

Proof. See Section 5.2.

Remark 2. The converse of Lemma 1 is not true. We provide a counterexample in Section S.2 of the Supplemental Material (Yokote 2020).

Intuitively, (5) states that the government intervention is minimal; it is allowed only if the resulting hires satisfy the constraints with equality. As suggested in standard market theory, the less government intervention there is, the more social welfare the market generates. This intuition is substantiated in Lemma 1 . We say that $\boldsymbol{t}$ induces $\boldsymbol{\mu}$ to be constrained efficient if $(\mu, t)$ satisfies (5).

A transfer policy $t$ is optimal if there exists a salary system $s$ and a matching $\mu$ such that the triple $(\mu, s, t)$ forms an uncompelled competitive equilibrium, $\mu$ is feasible, and $t$ induces $\mu$ to be constrained efficient.

\subsection{Main Theorems on Optimal Transfer Policies}

We characterize the optimal transfer policies and the corresponding equilibrium salaries as the minimizers of a Lagrange function. Together with Kojima et al.'s (2019) result, this characterization establishes the existence of optimal transfer policies. For each $h \in H$, we define $G_{h}: \mathbb{Z} \rightarrow \mathbb{Z}$ by

$$
G_{h}\left(t_{t}\right)= \begin{cases}-\underline{\delta}_{h} \cdot t_{h} & \text { if } t_{h} \geq 0 \\ -\bar{\delta}_{h} \cdot t_{h} & \text { if } t_{h}<0 .\end{cases}
$$

Fig. 1 shows a graphical description of ${ }^{14} G_{h}(\cdot)$.

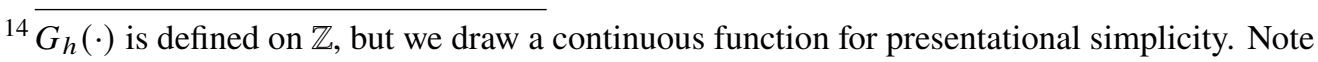
that this function has a "convex" shape, which is utilized in the proof of Theorem 3. 


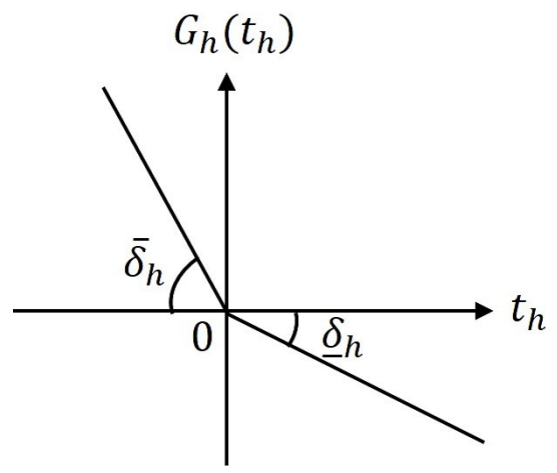

Fig. 1 Graphical description of $G_{h}(\cdot)$.

Set $G(t) \equiv \sum_{h \in H} G_{h}\left(t_{h}\right)$ for all $t \in \mathbb{Z}^{H}$. We define $F: \mathbb{Z}_{+}^{\Omega} \times \mathbb{Z}^{H} \rightarrow \mathbb{Z}$ by

$$
F(s, t)=\sum_{d \in D} V_{d}(s)+\sum_{h \in H} V_{h}(s, t)+G(t) \text { for all }(s, t) \in \mathbb{Z}_{+}^{\Omega} \times \mathbb{Z}^{H}
$$

Theorem 2 (Characterization of Optimal Transfer Policies and Equilibrium Salaries). Let $\left(s^{*}, t^{*}\right) \in \mathbb{Z}_{+}^{\Omega} \times \mathbb{Z}^{H}$. The following statements are equivalent:

(i) $\left(s^{*}, t^{*}\right)$ is a solution to

$$
\min _{s \in \mathbb{Z}_{+}^{\Omega}} \min _{t \in \mathbb{Z}^{H}} F(s, t)
$$

(ii) There exists a matching $\mu$ such that the triple $\left(\mu, s^{*}, t^{*}\right)$ forms an uncompelled competitive equilibrium, $\mu$ is feasible, and $t^{*}$ induces $\mu$ to be constrained efficient (i.e., (5) holds).

Proof. See Section 5.3.

Remark 3. Theorem 2 is silent about the existence of $\left(s^{*}, t^{*}\right) \in \mathbb{Z}_{+}^{\Omega} \times \mathbb{Z}^{H}$ satisfying (ii) (equivalently (i)), but this can be proven by combining existing results. First, as in the proof of Theorem 7 of Kojima et al. (2019), we consider a competitive equilibrium (a pair of a matching and a salary system) in the market where government-imposed feasibility collections are enforced (i.e., each hospital $h$ has a revenue function $\tilde{v}_{h}(\cdot)$ such that $\tilde{v}_{h}(A)=v_{h}(A)$ if $A \in \mathcal{F}_{h}$ and $\tilde{v}_{h}(A)=-\infty$ otherwise). Then, we can find an integer-valued competitive 
salary system $s^{*}$ and a corresponding matching ${ }^{15} \mu$. Following the proof of Lemma 7 of Kojima et al. (2019), we can construct an integer-valued transfer policy $t^{*}$ for which (5) holds.

In the proof, we apply Theorem 1 to the problem of maximizing the sum of the agents' utilities/profits under the following constraints:

- each doctor is matched to at most one hospital, and

- each hospital's hires satisfy the floor/ceiling constraints.

As will be detailed in Section 5.3.2 (sketch of the proof), we construct three independent maximization problems, where the assumptions in Theorem 1 hold, and aggregate the Lagrange functions in each problem into a single function. The resulting function corresponds to (7).

In (7), the first and second summations represent the doctors' total utility and the hospitals' total profit, respectively, while $G(t)$ coincides with the government surplus at optimal solutions. ${ }^{16}$ To see this point, let $h \in H$ and suppose that $t_{h} \geq 0$, i.e., the government subsidizes $h$. If $h$ hires exactly $\underline{\delta}_{h}$ workers, then the government expenditure is equal to $-\underline{\delta}_{h} \cdot t_{h}=G_{h}\left(t_{h}\right)$. If $h$ hires more than $\underline{\delta}_{h}$, then (5) implies that $t_{h}$ is equal to 0 . Namely, the government expenditure is $0=G_{h}\left(t_{h}\right)$. A parallel argument holds for $t_{h} \leq 0$ (i.e., in the case of taxation).

Finally, we discuss the algorithmic aspect of minimizing $F(\cdot, \cdot)$.

Theorem 3 ( $\mathrm{L}^{\natural}$-Convexity of the Lagrange Function). $F(\cdot, \cdot)$ is an $L^{\natural}$-convex function.

$\mathrm{L}^{\natural}$-convex function minimization algorithms have been studied extensively in the literature, and their upper bounds on the number of iterations have been revealed; see Murota (2003b), Kolmogorov and Shioura (2009) or Murota and Shioura (2014). ${ }^{17}$ As noted by Murota (2003b), complexity bounds are

15 This statement can be proven in several ways. For example, following Candogan et al. (2016), we can translate the market into the M-convex submodular flow problem and utilize the existence of integer-valued potential (see Theorem 9.16 of Murota 2003a). Alternatively, we can adopt the techniques in Fujishige and Tamura (2007).

16 This statement is formally proved in Claim 6 (see Section 5.3.1).

${ }^{17}$ Murota and Shioura (2014) demonstrate that the exact number of iterations can be obtained if the initial vector and the set of minimizers are known. Using this result, Murota et al. (2016) offer computational complexity results in an auction model. For a survey of algorithms, see Sections 7.3 and 8 of Murota (2016). 
polynomial in the dimension of the variable (which is equal to $(|D| \times|H|)+|H|$ in our model) and the size of the effective domain. In practice, we can restrict the size of the effective domain by setting sufficiently high salaries and sufficiently low/high transfers. Together with Theorems 2 and 3, we conclude that an optimal transfer policy can be calculated via an efficient algorithm. Section S.3 of the Supplemental Material (Yokote 2020) provides an example of how this algorithm proceeds.

\section{CONCLUDING REMARKS}

As pointed out by Gul and Stacchetti (1999), the job-matching model subsumes the auction model as a special case. Applying our approach to the auction model, the resulting Lagrange function corresponds to the Lyapunov function of Ausubel (2006) and Sun and Yang (2009). Since existing auction algorithms for heterogeneous commodities can be recast into minimization of the Lyapunov function (see Murota et al. 2016), we can view existing auctions as a process for finding a saddle point of the Lagrange function. For a formal analysis, see Section S.4 of the Supplemental Material (Yokote 2020).

It would be possible to extend our approach to the trading network model under quasi-linearity due to Hatfield et al. (2013). Hatfield et al. (2019) prove that the full substitutes condition imposed on preferences is equivalent to $\mathrm{M}^{\natural}$-concavity of the utility function, ${ }^{18}$ which enables the application of discrete convex analysis.

\section{THE APPENDICES}

This section contains all the proofs.

${ }^{18}$ To be precise, the agents have gross substitutes and complements (see Sun and Yang 2009) or a twisted $\mathrm{M}^{\natural}$-concave function (see Ikebe and Tamura 2015 and Section 3.5 of Murota 2016), which is essentially equivalent to an $\mathrm{M}^{\natural}$-concave function. 


\subsection{Proof of Theorem 1}

\subsubsection{Preliminaries}

For $x \in \mathbb{Z}^{K}$ and $A \subseteq K$, we define $x(A)=\sum_{k \in A} x_{k}$. For $X \subseteq \mathbb{R}^{K}$, we define the indicator function of $\boldsymbol{X}, \delta_{X}: \mathbb{R}^{K} \rightarrow \mathbb{Z} \cup\{-\infty\}$, by

$$
\delta_{X}(x)= \begin{cases}0 & \text { if } x \in X \\ -\infty & \text { otherwise }\end{cases}
$$

We say that $X \subseteq \mathbb{Z}^{K}$ with $X \neq \emptyset$ is an $\mathbf{M}^{\natural}$-convex set if $\delta_{X}(\cdot)$ is an $\mathbf{M}^{\natural}$-concave function. We say that $X \subseteq \mathbb{Z}^{K}$ with $X \neq \emptyset$ is an $\mathbf{L}^{\natural}$-convex set if $\delta_{X}(\cdot)$ is an $\mathrm{L}^{\natural}$-concave function.

For $f: \mathbb{Z}^{K} \rightarrow \mathbb{Z} \cup\{-\infty\}$ and $x^{*} \in \operatorname{dom} f$, we define the supergradient of $f$ at $x^{*}($ in $\mathbb{Z})$ by

$$
\partial_{\mathbb{Z}} f\left(x^{*}\right)=\left\{\hat{x} \in \mathbb{Z}^{K}: f\left(x^{*}\right)+\hat{x} \cdot\left(x-x^{*}\right) \geq f(x) \text { for all } x \in \mathbb{Z}^{K}\right\} .
$$

For $f: \mathbb{R}^{K} \rightarrow \mathbb{R} \cup\{-\infty\}$ and $x^{*} \in \operatorname{dom} f$, we define the supergradient of $\boldsymbol{f}$ at $x^{*}$ (in $\left.\mathbb{R}\right)$ by

$$
\partial_{\mathbb{R}} f\left(x^{*}\right)=\left\{\hat{x} \in \mathbb{R}^{K}: f\left(x^{*}\right)+\hat{x} \cdot\left(x-x^{*}\right) \geq f(x) \text { for all } x \in \mathbb{R}^{K}\right\} .
$$

Let $\mathcal{K} \subseteq 2^{K} \backslash\{\emptyset\}$. We define the cone generated by $\left(\mathbb{1}^{A}\right)_{A \in \mathcal{K}}$ (in $\mathbb{Z}$ ) by

$$
\text { cone }_{\mathbb{Z}}(\mathcal{K})=\left\{x \in \mathbb{Z}^{K}: x=\sum_{A \in \mathcal{K}} \lambda_{A} \cdot \mathbb{1}^{A} \text { for some }\left(\lambda_{A}\right)_{A \in \mathcal{K}} \in \mathbb{Z}_{+}^{\mathcal{K}}\right\} .
$$

We define the cone generated by $\left(\mathbb{1}^{A}\right)_{A \in \mathcal{K}}($ in $\mathbb{R})$ by

$$
\operatorname{cone}_{\mathbb{R}}(\mathcal{K})=\left\{x \in \mathbb{R}^{K}: x=\sum_{A \in \mathcal{K}} \lambda_{A} \cdot \mathbb{1}^{A} \text { for some }\left(\lambda_{A}\right)_{A \in \mathcal{K}} \in \mathbb{R}_{+}^{\mathcal{K}}\right\} .
$$

For $X \subseteq \mathbb{Z}^{K}$, let $\bar{X} \subseteq \mathbb{R}^{K}$ denote the convex hull of $X$ and $X^{\circ}$ denote the polar of $X$, i.e., $X^{\circ}=\left\{y \in \mathbb{Z}^{K}: y \cdot x \leq 0\right.$ for all $\left.x \in X\right\}$. For $X, Y \subseteq \mathbb{R}^{K}$, we define

$$
-X=\left\{x \in \mathbb{R}^{K}:-x \in X\right\}, X-Y=\left\{x-y \in \mathbb{R}^{K}: x \in X, y \in Y\right\} .
$$

The following theorem is essential to the proof. 
Theorem 4 (Murota 2003a, Theorem 8.17 ( $\mathrm{M}^{\natural}$-concave intersection theorem)). For $\mathbf{M}^{\natural}$-concave functions $f_{1}, f_{2}: \mathbb{Z}^{K} \rightarrow \mathbb{Z} \cup\{-\infty\}$ and a point $x^{*} \in \operatorname{dom} f_{1} \cap$ $\operatorname{dom} f_{2}$, we have

$$
f_{1}\left(x^{*}\right)+f_{2}\left(x^{*}\right) \leq f_{1}(x)+f_{2}(x) \text { for all } x \in \mathbb{Z}^{K}
$$

if and only if there exists $\hat{x} \in \mathbb{Z}^{K}$ such that

$$
\hat{x} \in \partial_{\mathbb{Z}} f_{1}\left(x^{*}\right) \text { and }-\hat{x} \in \partial_{\mathbb{Z}} f_{2}\left(x^{*}\right) .
$$

Theorem 5 (Murota 2003a, (5.8) (Convexity in intersection for $\mathrm{L}^{\natural}$-convex sets)). For $\mathrm{L}^{\natural}$-convex sets $X_{1}, X_{2} \subseteq \mathbb{Z}^{K}$, we have

$$
\overline{X_{1}} \cap \overline{X_{2}} \neq \emptyset \Longrightarrow X_{1} \cap X_{2} \neq \emptyset \text {. }
$$

Claim 1. Let $\mathcal{K} \subseteq 2^{K} \backslash\{\emptyset\}$ with $\mathcal{K} \neq \emptyset$ be a hierarchy and $\left(\lambda_{A}\right)_{A \in \mathcal{K}} \in \mathbb{Z}^{\mathcal{K}}$. Then, the set

$$
X=\left\{x \in \mathbb{Z}^{K}: x(A) \leq \lambda_{A} \text { for all } A \in \mathcal{K}\right\}
$$

is an $\mathrm{M}^{\natural}$-convex set.

Proof. For each $A \in \mathcal{K}$, we define $\varphi_{A}: \mathbb{Z} \rightarrow \mathbb{Z} \cup\{-\infty\}$ by

$$
\varphi_{A}(x)= \begin{cases}0 & \text { if } x \leq \lambda_{A}, \\ -\infty & \text { otherwise. }\end{cases}
$$

We define $\Phi: \mathbb{Z}^{K} \rightarrow \mathbb{Z} \cup\{-\infty\}$ by

$$
\Phi(x)=\sum_{A \in \mathcal{K}} \varphi_{A}(x(A)) \text { for all } x \in \mathbb{Z}^{K} .
$$

Then, by Note 6.11 of Murota (2003a), $\Phi(\cdot)$ is an $M^{\natural}$-concave function. By Proposition 6.29 of Murota (2003a), the set of maximizers of $\Phi(\cdot)$ is an $\mathrm{M}^{\natural}$-convex set. As $X$ is the set of maximizers of $\Phi(\cdot)$, we obtain the desired condition.

Remark 4. To develop some intuition for Claim 1, we provide a 2-dimentional case example. Let $K=\left\{k_{1}, k_{2}\right\}$ and $\mathcal{K}=\left\{\left\{k_{1}\right\},\left\{k_{2}\right\},\left\{k_{1}, k_{2}\right\}\right\}$. Set $\lambda_{\left\{k_{1}\right\}}=$ $5, \lambda_{\left\{k_{2}\right\}}=6, \lambda_{\left\{k_{1}, k_{2}\right\}}=8$. Then, the set $X$ defined by (9) is described by the shaded area in Fig. 2. 


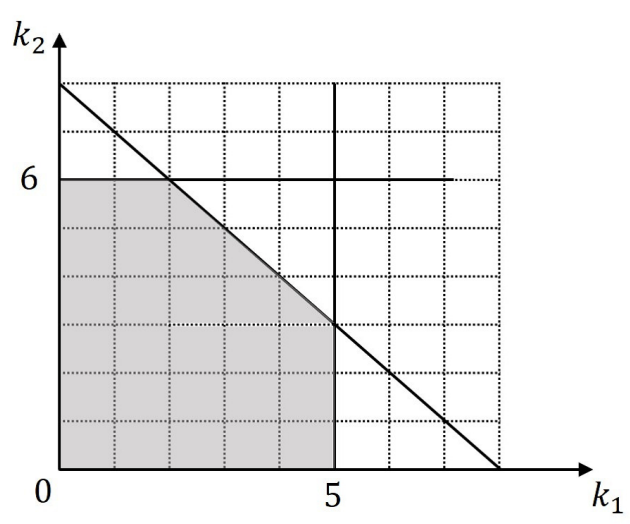

Fig. 2 Graphical descrption of $X$.

One easily verifies that this set is $\mathrm{M}^{\natural}$-convex.

Claim 1 can also be proven by using the transformation of $\mathrm{M}^{\natural}$-convexity by a network. A tree structure associated with a laminar family forms a network flow and the upper bounds $\left(\lambda_{A}\right)_{A \in \mathcal{K}}$ can be incorporated into the costs of flow. Then, Theorem 9.26 of Murota (2003a) establishes that $X$ is an $\mathbf{M}^{\natural}$-convex set. $^{19}$

Claim 2. Let $\mathcal{K} \subseteq 2^{K} \backslash\{\emptyset\}$ with $\mathcal{K} \neq \emptyset$ be a hierarchy. Then, the set

$$
\operatorname{cone}_{\mathbb{R}}(\mathcal{K}) \cap \mathbb{Z}^{K}
$$

is an $\mathrm{L}^{\natural}$-convex set.

Proof. This theorem follows from Claim 1 and the discrete conjugacy theorem (see Murota 2003a, Theorem 8.12).

Claim 3. Let $\mathcal{K} \subseteq 2^{K} \backslash\{\emptyset\}$. Then,

$$
\overline{\operatorname{cone}_{\mathbb{Z}}(\mathcal{K})}=\text { cone }_{\mathbb{R}}(\mathcal{K}) .
$$

Proof. If $\mathcal{K}=\emptyset$, then the claim trivially holds. Suppose that $\mathcal{K} \neq \emptyset$. For each $\lambda \in \mathbb{Z}$ with $\lambda>0$, let $\Delta^{\lambda}$ denote the $\lambda$-dimensional unit simplex.

Proof of $\subseteq$ : Let $x \in \overline{\text { cone }_{\mathbb{Z}}(\mathcal{K})}$. By Carathéodory's theorem, there exist $x_{j} \in \operatorname{cone}_{\mathbb{Z}}(\mathcal{K}), j=1, \ldots,|K|+1$, and $\alpha \in \Delta^{|K|}$ such that $x=\sum_{j} \alpha_{j} x_{j}$. For

${ }_{19}$ The author thanks a referee for pointing out this proof. 
each $x_{j} \in$ cone $_{\mathbb{Z}}(\mathcal{K})$, there exists $\left(\lambda_{A, j}\right)_{A \in \mathcal{K}} \in \mathbb{Z}_{+}^{\mathcal{K}}$ such that $x_{j}=\sum_{A \in \mathcal{K}} \lambda_{A, j} \mathbb{1}^{A}$. Then,

$$
x=\sum_{j=1}^{|K|+1} \alpha_{j} \sum_{A \in \mathcal{K}} \lambda_{A, j} \mathbb{1}^{A}=\sum_{A \in \mathcal{K}} \sum_{j=1}^{|K|+1}\left(\alpha_{j} \cdot \lambda_{A, j}\right) \mathbb{1}^{A} .
$$

Hence, $x \in$ cone $_{\mathbb{R}}(\mathcal{K})$.

Proof of $\supseteq$ : Let $x \in$ cone $_{\mathbb{R}}(\mathcal{K})$. Then, there exists $\left(\lambda_{A}\right)_{A \in \mathcal{K}} \in \mathbb{R}_{+}^{\mathcal{K}}$ such that $x=\sum_{A \in \mathcal{K}} \lambda_{A} \chi_{A}$. By $\mathbb{R}_{+}^{\mathcal{K}}=\overline{\mathbb{Z}_{+}^{\mathcal{K}}}$ and Carathéodory's theorem, there exist $\left(z_{A, j}\right)_{A \in \mathcal{K}} \in \mathbb{Z}_{+}^{\mathcal{K}}, j=1, \ldots,|\mathcal{K}|+1$, and $\alpha \in \Delta^{|\mathcal{K}|}$ such that $\lambda_{A}=\sum_{j} \alpha_{j} z_{A, j}$ for all $A \in \mathcal{K}$. Then,

$$
x=\sum_{A \in \mathcal{K}}\left(\sum_{j=1}^{|\mathcal{K}|+1} \alpha_{j} z_{A, j}\right) \mathbb{1}^{A}=\sum_{j=1}^{|\mathcal{K}|+1} \alpha_{j} \sum_{A \in \mathcal{K}} z_{A, j} \mathbb{1}^{A} .
$$

Hence, $x \in \overline{\text { cone }_{\mathbb{Z}}(\mathcal{K})}$.

Claim 4. For any hierarchy $\mathcal{K} \subseteq 2^{K} \backslash\{\emptyset\}$,

$$
\text { cone }_{\mathbb{R}}(\mathcal{K}) \cap \mathbb{Z}^{K}=\text { cone }_{\mathbb{Z}}(\mathcal{K}) .
$$

Proof. One easily verifies that $\supseteq$ holds. We prove $\subseteq$ by induction on $|\mathcal{K}|$.

Induction base: Suppose $|\mathcal{K}|=1$. Let $x \in \operatorname{cone}_{\mathbb{R}}(\mathcal{K}) \cap \mathbb{Z}^{K}$. Assuming $\mathcal{K}=\{A\}, x=\lambda_{A} \cdot \mathbb{1}^{A}$ for some $\lambda_{A} \in \mathbb{R}_{+}$. Since $x \in \mathbb{Z}^{K}$, we have $\lambda_{A} \in \mathbb{Z}_{+}$. Hence, $x \in$ cone $_{\mathbb{Z}}(\mathcal{K})$.

Induction step: Suppose the claim holds for $|\mathcal{K}|=t$ and we prove the claim for $|\mathcal{K}|=t+1$, where $t \geq 1$.

Let $x \in$ cone $_{\mathbb{R}}(\mathcal{K}) \cap \mathbb{Z}^{K}$ and $A^{\prime} \in \mathcal{K}$. Then, there exists $\left(\lambda_{A}\right)_{A \in \mathcal{K}} \in \mathbb{R}_{+}^{\mathcal{K}}$ such that

$$
x=\sum_{A \in \mathcal{K} \backslash\left\{A^{\prime}\right\}} \lambda_{A} \cdot \mathbb{1}^{A}+\lambda_{A^{\prime}} \cdot \mathbb{1}^{A^{\prime}} .
$$

This implies that

$$
\text { cone }_{\mathbb{R}}\left(\mathcal{K} \backslash\left\{A^{\prime}\right\}\right) \cap\left(\{x\}-\text { cone }_{\mathbb{R}}\left(\left\{A^{\prime}\right\}\right)\right) \neq \emptyset .
$$

By Claim 3,

$$
\operatorname{cone}_{\mathbb{R}}\left(\mathcal{K} \backslash\left\{A^{\prime}\right\}\right)=\overline{\text { cone }_{\mathbb{Z}}\left(\mathcal{K} \backslash\left\{A^{\prime}\right\}\right)}
$$


We also have

$$
\begin{aligned}
\{x\}-\text { cone }_{\mathbb{R}}\left(\left\{A^{\prime}\right\}\right) & =\{x\}-\overline{\text { cone }_{\mathbb{Z}}\left(\left\{A^{\prime}\right\}\right)} \\
& =\overline{\{x\}-\text { cone }_{\mathbb{Z}}\left(\left\{A^{\prime}\right\}\right)},
\end{aligned}
$$

where the first equality follows from Claim 3 and the second equality follows from Proposition 3.17(4) of Murota (2003a).

By (10)-(12),

$$
\overline{\text { cone }_{\mathbb{Z}}\left(\mathcal{K} \backslash\left\{A^{\prime}\right\}\right)} \cap \overline{\{x\}-\text { cone }_{\mathbb{Z}}\left(\left\{A^{\prime}\right\}\right)} \neq \emptyset .
$$

As $\mathcal{K} \backslash\left\{A^{\prime}\right\}$ is a hierarchy, by the induction hypothesis and Claim 2, it holds that cone $_{\mathbb{Z}}\left(\mathcal{K} \backslash\left\{A^{\prime}\right\}\right)$ is $\mathrm{L}^{\natural}$-convex. One easily verifies that $\{x\}-$ cone $_{\mathbb{Z}}\left(\left\{A^{\prime}\right\}\right)$ is also $\mathrm{L}^{\natural}$-convex. By Theorem 5,

$$
\text { cone }_{\mathbb{Z}}\left(\mathcal{K} \backslash\left\{A^{\prime}\right\}\right) \cap\left(\{x\}-\text { cone }_{\mathbb{Z}}\left(\left\{A^{\prime}\right\}\right)\right) \neq \emptyset .
$$

This implies that $x \in$ cone $_{\mathbb{Z}}(\mathcal{K})$.

\subsubsection{Proof of $(i i) \Rightarrow(i)$}

We mimic the proof of Karush-Kuhn-Tucker's theorem (for continuous settings) by Tiel (1984, p.103).

By the latter inequality in (1), $L\left(x^{*}, \lambda^{*}\right) \leq L\left(x^{*}, \lambda\right)$ for all $\lambda \in \mathbb{Z}_{+}^{q}$. Together with (2),

$$
\begin{aligned}
\sum_{j=1}^{q} \lambda_{j}^{*} g_{j}\left(x^{*}\right) & \leq \sum_{j=1}^{q} \lambda_{j} g_{j}\left(x^{*}\right) \text { for all } \lambda \in \mathbb{Z}_{+}^{q}, \\
0 & \leq \sum_{j=1}^{q}\left(\lambda_{j}-\lambda_{j}^{*}\right) g_{j}\left(x^{*}\right) \text { for all } \lambda \in \mathbb{Z}_{+}^{q} .
\end{aligned}
$$

Since (13) holds for all $\lambda \in \mathbb{Z}_{+}^{q}$,

$$
g_{j}\left(x^{*}\right) \geq 0 \text { for all } j=1, \ldots, q .
$$

Letting $\lambda=\mathbf{0}$ in (13),

$$
\sum_{j=1}^{q} \lambda_{j}^{*} g_{j}\left(x^{*}\right) \leq 0 .
$$


Combining this inequality with (14) yields

$$
\sum_{j=1}^{q} \lambda_{j}^{*} g_{j}\left(x^{*}\right)=0
$$

This equation and the former inequality in (1) imply

$$
f(x)+\sum_{j=1}^{q} \lambda_{j}^{*} g_{j}(x) \leq f\left(x^{*}\right) \text { for all } x \in \mathbb{Z}^{K} .
$$

This means that $f(x) \leq f\left(x^{*}\right)$ whenever $g_{j}(x) \geq 0$ for all $j=1, \ldots, q$. Together with (14), we obtain the desired condition.

\subsubsection{Proof of $(i) \Rightarrow$ (ii)}

By assumption, for all $j=1, \ldots, q$, there exist $a_{j} \in \mathbb{Z}$ and $A_{j} \subseteq K$ with $A_{j} \neq \emptyset$ such that

$$
g_{j}(x)=a_{j}-x\left(A_{j}\right) \text { for all } x \in \mathbb{Z}^{K} .
$$

Our purpose is to find $\lambda^{*} \in \mathbb{Z}_{+}^{q}$ such that $\left(x^{*}, \lambda^{*}\right)$ satisfies (1). Suppose $A_{j}=A_{j^{\prime}}$ for some $j, j^{\prime} \in\{1, \ldots, q\}$ with $a_{j} \leq a_{j^{\prime}}$. Then, $g_{j^{\prime}}(\cdot)$ is a redundant constraint function. In the proof below, we can ignore such $j^{\prime}$ by letting $\lambda_{j^{\prime}}^{*}=0$. Hence, w.l.o.g., we assume

$$
A_{j} \neq A_{j^{\prime}} \text { for all } j, j^{\prime} \in\{1, \ldots, q\} \text {. }
$$

Set $C=\left\{x \in \mathbb{Z}^{K}: g_{j}(x) \geq 0\right.$ for all $\left.j=1, \ldots, q\right\}$. By Claim $1, \delta_{C}(\cdot)$ is an $\mathrm{M}^{\natural}$-concave function. Since $x^{*}$ is a solution to the maximization problem under constraints,

$$
f\left(x^{*}\right)+\delta_{C}\left(x^{*}\right) \geq f(x)+\delta_{C}(x) \text { for all } x \in \mathbb{Z}^{K} .
$$

By Theorem 4, there exists $\hat{x} \in \mathbb{Z}^{K}$ such that

$$
\begin{array}{r}
\hat{x} \in \partial_{\mathbb{Z}} f\left(x^{*}\right), \\
-\hat{x} \in \partial_{\mathbb{Z}} \delta_{C}\left(x^{*}\right) .
\end{array}
$$


By (15) and the definition of a supergradient, $-\hat{x} \in \partial_{\mathbb{R}} \delta_{\bar{C}}\left(x^{*}\right)$. As recognized in the literature on convex analysis (see, for example, Rockafellar (1970), Section $23),-\partial_{\mathbb{R}} \delta_{\bar{C}}\left(x^{*}\right)$ is the normal cone to $\bar{C}$ at $x^{*}$. i.e.,

$$
-\partial_{\mathbb{R}} \delta_{\bar{C}}\left(x^{*}\right)=\left\{y \in \mathbb{R}^{K}: y \cdot\left(x-x^{*}\right) \leq 0 \text { for all } x \in \bar{C}\right\} .
$$

By Proposition 5.2.4 of Hiriart-Urruty and Lemaréchal (2001), the normal cone is the polar of the tangent cone. This fact and (15) imply $\hat{x} \in$ cone $_{\mathbb{R}}(\mathcal{K})$, where

$$
\mathcal{K}=\left\{A \subseteq K: A=A_{j} \text { for some } j \in\{1, \ldots, q\} \text { with } g_{j}\left(x^{*}\right)=0\right\}
$$

Since $\mathcal{K}$ is a hierarchy, by Claim $4, \hat{x} \in \operatorname{cone}_{\mathbb{Z}}(\mathcal{K})$. Hence, there exists $\left(\lambda_{A}^{*}\right)_{A \in \mathcal{K}} \in \mathbb{Z}_{+}^{\mathcal{K}}$ such that

$$
\hat{x}=\sum_{A \in \mathcal{K}} \lambda_{A}^{*} \mathbb{1}^{A} .
$$

For each $j \in\{1, \ldots, q\}$ with $g_{j}\left(x^{*}\right)=0$, set $\lambda_{j}^{*}=\lambda_{A_{j}}^{*}$ for $A_{j} \in \mathcal{K}$. For each $j \in\{1, \ldots, q\}$ with $g_{j}\left(x^{*}\right)>0$, set $\lambda_{j}^{*}=0$.

We prove that $\left(x^{*}, \lambda^{*}\right)$ is a saddle point of $L(\cdot, \cdot)$ defined by (2). We first fix $\lambda^{*}$ and regard $L\left(\cdot, \lambda^{*}\right)$ as a function on $\mathbb{R}^{K}$. Then,

$$
\begin{aligned}
\partial_{\mathbb{Z}} L\left(x^{*}, \lambda^{*}\right) & =\partial_{\mathbb{Z}}\left(f\left(x^{*}\right)+\sum_{j=1}^{q} \lambda_{j}^{*} g_{j}\left(x^{*}\right)\right) \\
& =\partial_{\mathbb{Z}} f\left(x^{*}\right)-\left\{\sum_{j=1}^{q} \lambda_{j}^{*} \mathbb{1}^{A_{j}}\right\} \\
& \ni \hat{x}-\hat{x} \\
& =0,
\end{aligned}
$$

where the second equality, decomposition of superdifferential, follows from the fact that $g_{j}(\cdot), j=1, \ldots, q$, are affine functions. ${ }^{20}$ This means that $L\left(\cdot, \lambda^{*}\right)$ is maximized at $x^{*}$.

${ }^{20}$ More generally, decomposition of superdifferential holds for two $\mathbf{M}^{\natural}$-concave functions; see Theorem 8.35 of Murota (2003a). 
Next fix $x^{*}$ and regard $L\left(x^{*}, \cdot\right)$ as a function on $\mathbb{Z}_{+}^{q}$. As $x^{*}$ satisfies the constraints, $g_{j}\left(x^{*}\right) \geq 0$ for all $j=1, \ldots, q$. Hence, for any $\lambda \in \mathbb{Z}_{+}^{q}$,

$$
\sum_{j=1}^{q} \lambda_{j} g_{j}\left(x^{*}\right) \geq 0
$$

Moreover, by the construction of $\lambda^{*}$,

$$
\sum_{j=1}^{q} \lambda_{j}^{*} g_{j}\left(x^{*}\right)=0
$$

We conclude

$$
\begin{array}{r}
L\left(x^{*}, \lambda\right)=f\left(x^{*}\right)+\sum_{j=1}^{q} \lambda_{j} g_{j}\left(x^{*}\right) \geq f\left(x^{*}\right)+\sum_{j=1}^{q} \lambda_{j}^{*} g_{j}\left(x^{*}\right)=L\left(x^{*}, \lambda^{*}\right) \\
\text { for all } \lambda \in \mathbb{Z}_{+}^{q},
\end{array}
$$

where the inequality follows from (16) and (17). Finally, the complementary slackness follows from the construction of $\lambda^{*}$.

\subsection{Proof of Lemma 1}

We prove a claim.

Claim 5. Let $\mu$ be a matching and $s \in \mathbb{Z}_{+}^{\Omega}$. Then,

$$
\begin{aligned}
& \sum_{d \in D} v_{d}[s](\mu(d))+\sum_{h \in H} v_{h}[s, t](\mu(h)) \\
= & \sum_{d \in D} v_{d}(\mu(d))+\sum_{h \in H} v_{h}(\mu(h))+\sum_{h \in H}|\mu(h)| \cdot t_{h} .
\end{aligned}
$$

Proof. Since $\mu$ is a matching,

$$
\sum_{d \in D} s_{(d, h)}=\sum_{h \in H} \sum_{d \in \mu(h)} s_{(d, h)} .
$$


Then,

$$
\begin{aligned}
& \sum_{d \in D} v_{d}[s](\mu(d))+\sum_{h \in H} v_{h}[s, t](\mu(h)) \\
= & \sum_{d \in D}\left\{v_{d}(\mu(d))+s_{(d, h)}\right\}+\sum_{h \in H}\left\{v_{h}(\mu(h))-\sum_{d \in \mu(h)} s_{(d, h)}+|\mu(h)| \cdot t_{h}\right\} \\
= & \sum_{d \in D} v_{d}(\mu(d))+\sum_{h \in H} v_{h}(\mu(h))+\sum_{h \in H}|\mu(h)| \cdot t_{h},
\end{aligned}
$$

where the second equality follows from (18).

We start the proof of Lemma 1. Let $(\mu, s, t)$ be an uncompelled competitive equilibrium such that $\mu$ is feasible and the pair $(\mu, t)$ satisfies (5). Let $\mu^{\prime}$ be an arbitrary feasible matching. Then,

$$
\begin{aligned}
& \sum_{d \in D} v_{d}(\mu(d))+\sum_{h \in H} v_{h}(\mu(h)) \\
= & \sum_{d \in D} v_{d}[s](\mu(d))+\sum_{h \in H} v_{h}[s, t](\mu(h))-\sum_{h \in H}|\mu(h)| \cdot t_{h} \\
\geq & \sum_{d \in D} v_{d}[s]\left(\mu^{\prime}(d)\right)+\sum_{h \in H} v_{h}[s, t]\left(\mu^{\prime}(h)\right)-\sum_{h \in H}|\mu(h)| \cdot t_{h} \\
= & \sum_{d \in D} v_{d}\left(\mu^{\prime}(d)\right)+\sum_{h \in H} v_{h}\left(\mu^{\prime}(h)\right)+\sum_{h \in H}\left|\mu^{\prime}(h)\right| \cdot t_{h}-\sum_{h \in H}|\mu(h)| \cdot t_{h} \\
= & \sum_{d \in D} v_{d}\left(\mu^{\prime}(d)\right)+\sum_{h \in H} v_{h}\left(\mu^{\prime}(h)\right) \\
+ & \sum_{h \in H: t_{h}>0}\left(\left|\mu^{\prime}(h)\right|-|\mu(h)|\right) \cdot t_{h}+\sum_{h \in H: t_{h}<0}\left(\left|\mu^{\prime}(h)\right|-|\mu(h)|\right) \cdot t_{h},
\end{aligned}
$$

where the first equality follows from Claim 5 , the first inequality follows from the fact that $(\mu, s, t)$ is an uncompelled competitive equilibrium, and the second equality follows from Claim 5. By (5) for $(\mu, t)$ and feasibility of $\mu^{\prime}$, for any $h \in H$,

$$
\begin{aligned}
& t_{h}>0 \Longrightarrow|\mu(h)|=\underline{\delta}_{h} \leq\left|\mu^{\prime}(h)\right| \Longrightarrow\left(\left|\mu^{\prime}(h)\right|-|\mu(h)|\right) \cdot t_{h} \geq 0, \\
& t_{h}<0 \Longrightarrow|\mu(h)|=\bar{\delta}_{h} \geq\left|\mu^{\prime}(h)\right| \Longrightarrow\left(\left|\mu^{\prime}(h)\right|-|\mu(h)|\right) \cdot t_{h} \geq 0 .
\end{aligned}
$$


It follows that

$$
\sum_{h \in H: t_{h}>0}\left(\left|\mu^{\prime}(h)\right|-|\mu(h)|\right) \cdot t_{h}+\sum_{h \in H: t_{h}<0}\left(\left|\mu^{\prime}(h)\right|-|\mu(h)|\right) \cdot t_{h} \geq 0 .
$$

Together with (19), we conclude

$$
\begin{aligned}
\mathbf{V}(\mu) & =\sum_{d \in D} v_{d}(\mu(d))+\sum_{h \in H} v_{h}(\mu(h)) \\
& \geq \sum_{d \in D} v_{d}\left(\mu^{\prime}(d)\right)+\sum_{h \in H} v_{h}\left(\mu^{\prime}(h)\right) \\
& =\mathbf{V}\left(\mu^{\prime}\right) .
\end{aligned}
$$

\subsection{Proof of Theorem 2}

\subsubsection{Proof of $($ ii $) \Rightarrow(i)$}

We prove a claim.

Claim 6. Suppose that for a matching $\mu$ and $t \in \mathbb{Z}^{H}$, (5) holds. Then, for any $h \in H$,

$$
-|\mu(h)| \cdot t_{h}=G_{h}\left(t_{h}\right) .
$$

Proof. Let $h \in H$ be arbitrarily chosen. We consider three cases.

Case 1: If $t_{h}=0$, then $-|\mu(h)| \cdot t_{h}=0=G_{h}\left(t_{h}\right)$.

Case 2: If $t_{h}>0,(5)$ implies $|\mu(h)|=\underline{\delta}_{h}$. Then, $-|\mu(h)| \cdot t_{h}=-\underline{\delta}_{h} \cdot t_{h}=$ $G_{h}\left(t_{h}\right)$.

Case 3: If $t_{h}<0$, (5) implies $|\mu(h)|=\bar{\delta}_{h}$. Then, $-|\mu(h)| \cdot t_{h}=-\bar{\delta}_{h} \cdot t_{h}=$ $G_{h}\left(t_{h}\right)$.

We start the proof of (ii) $\Rightarrow$ (i). Let $\left(s^{*}, t^{*}\right) \in \mathbb{Z}_{+}^{\Omega} \times \mathbb{Z}^{H}$. Suppose that there exists a matching $\mu$ that satisfies the conditions in the statement of (ii). By Theorem 3, which we establish later, $L(\cdot, \cdot)$ is an $L^{\natural}$-convex function. By Theorem 7.14 of Murota (2003a) (known as the L-optimality criterion), it suffices to prove that, for any $\Psi \subseteq \Omega$ and $A \subseteq H$,

$$
\begin{aligned}
& F\left(s^{*}, t^{*}\right) \leq F\left(s^{*}+\mathbb{1}^{\Psi}, t^{*}+\mathbb{1}^{A}\right), \\
& F\left(s^{*}, t^{*}\right) \leq F\left(s^{*}-\mathbb{1}^{\Psi}, t^{*}-\mathbb{1}^{A}\right) .
\end{aligned}
$$


Let $\Psi \subseteq \Omega$ and $A \subseteq H$ be arbitrarily chosen. We prove (20) and skip the proof of (21) which can be obtained analogously.

The left-hand side of (20) is rewritten as follows:

$$
\begin{aligned}
F\left(s^{*}, t^{*}\right) & =\sum_{d \in D} V_{d}\left(s^{*}\right)+\sum_{h \in H} V_{h}\left(s^{*}, t^{*}\right)+G\left(t^{*}\right) \\
& =\sum_{d \in D} v_{d}\left[s^{*}\right](\mu(d))+\sum_{h \in H} v_{h}\left[s^{*}, t^{*}\right](\mu(h))+G\left(t^{*}\right) \\
& =\sum_{d \in D} v_{d}(\mu(d))+\sum_{h \in H} v_{h}(\mu(h))+\sum_{h \in H}|\mu(h)| \cdot t_{h}^{*}+\sum_{h \in H} G_{h}\left(t_{h}^{*}\right),
\end{aligned}
$$

where the second equality follows from the fact that $\left(\mu, s^{*}, t^{*}\right)$ is an uncompelled competitive equilibrium and the third equality follows from Claim 5. By Claim 6 ,

$$
\sum_{h \in H}|\mu(h)| \cdot t_{h}^{*}+\sum_{h \in H} G_{h}\left(t_{h}^{*}\right)=0 .
$$

Substituting (23) into (22) we obtain

$$
F\left(s^{*}, t^{*}\right)=\sum_{d \in D} v_{d}(\mu(d))+\sum_{h \in H} v_{h}(\mu(h)) .
$$

The right-hand side of (20) is rewritten as follows:

$$
\begin{aligned}
& F\left(s^{*}+\mathbb{1}^{\Psi}, t^{*}+\mathbb{1}^{A}\right) \\
& =\sum_{d \in D} V_{d}\left(s^{*}+\mathbb{1}^{\Psi}\right)+\sum_{h \in H} V_{h}\left(s^{*}+\mathbb{1}^{\Psi}, t^{*}+\mathbb{1}^{A}\right)+G\left(t^{*}+\mathbb{1}^{A}\right) \\
& \geq \sum_{d \in D} v_{d}\left[s^{*}+\mathbb{1}^{\Psi}\right](\mu(d))+\sum_{h \in H} v_{h}\left[s^{*}+\mathbb{1}^{\Psi}, t^{*}+\mathbb{1}^{A}\right](\mu(h))+G\left(t^{*}+\mathbb{1}^{A}\right) \\
& =\sum_{d \in D} v_{d}(\mu(d))+\sum_{h \in H} v_{h}(\mu(h))+\sum_{h \in H}|\mu(h)| \cdot\left(t_{h}^{*}+\mathbb{1}_{h}^{A}\right)+\sum_{h \in H} G_{h}\left(t_{h}^{*}+\mathbb{1}_{h}^{A}\right),
\end{aligned}
$$

where the inequality follows from the definition of indirect utility functions and the second equality follows from Claim 5. Together with (24), in order to prove (20), it suffices to prove that

$$
\sum_{h \in H}|\mu(h)| \cdot\left(t_{h}^{*}+\mathbb{1}_{h}^{A}\right)+\sum_{h \in H} G_{h}\left(t_{h}^{*}+\mathbb{1}_{h}^{A}\right) \geq 0 .
$$


Let $h \in H$ be arbitrarily chosen. To prove the above inequality, it suffices to prove that

$$
|\mu(h)| \cdot\left(t_{h}^{*}+\mathbb{1}_{h}^{A}\right)+G_{h}\left(t_{h}^{*}+\mathbb{1}_{h}^{A}\right) \geq 0 .
$$

Proof of (25): If $h \notin A$, then $\mathbb{1}_{h}^{A}=0$. By Claim 6, the inequality holds with equality. Suppose that $h \in A$. We consider two cases.

Case 1: Suppose $t_{h}^{*}<0$. By (5),

$$
|\mu(h)|=\bar{\delta}_{h} .
$$

By the definition of $G_{h}(\cdot)$,

$$
G_{h}\left(t_{h}^{*}\right)-G_{h}\left(t_{h}^{*}+\mathbb{1}_{h}^{A}\right)=\bar{\delta}_{h} .
$$

Then,

$$
\begin{aligned}
|\mu(h)| \cdot\left(t_{h}^{*}+\mathbb{1}_{h}^{A}\right)+G_{h}\left(t_{h}^{*}+\mathbb{1}_{h}^{A}\right) & =-G_{h}\left(t_{h}^{*}\right)+|\mu(h)| \cdot \mathbb{1}_{h}^{A}+G_{h}\left(t_{h}^{*}+\mathbb{1}_{h}^{A}\right) \\
& =-\bar{\delta}_{h}+|\mu(h)| \cdot \mathbb{1}_{h}^{A} \\
& =-\bar{\delta}_{h}+\bar{\delta}_{h} \\
& =0
\end{aligned}
$$

where the first equality follows from Claim 6, the second equality follows from (27), and the third equality follows from (26).

Case 2: Suppose $t_{h}^{*} \geq 0$. By the definition of $G_{h}(\cdot)$,

$$
G_{h}\left(t_{h}^{*}\right)-G_{h}\left(t_{h}^{*}+\mathbb{1}_{h}^{A}\right)=\underline{\delta}_{h} .
$$

Then,

$$
\begin{aligned}
|\mu(h)| \cdot\left(t_{h}^{*}+\mathbb{1}_{h}^{A}\right)+G_{h}\left(t_{h}^{*}+\mathbb{1}_{h}^{A}\right) & =-G_{h}\left(t_{h}^{*}\right)+|\mu(h)| \cdot \mathbb{1}_{h}^{A}+G_{h}\left(t_{h}^{*}+\mathbb{1}_{h}^{A}\right) \\
& =-\underline{\delta}_{h}+|\mu(h)| \cdot \mathbb{1}_{h}^{A} \\
& \geq-\underline{\delta}_{h}+\underline{\delta}_{h} \\
& =0,
\end{aligned}
$$

where the first equality follows from Claim 6, the second equality follows from (28), and the inequality follows from feasibility of $\mu$. 


\subsubsection{Sketch of the proof of $(i) \Rightarrow$ (ii)}

We consider three maximization problems. In each problem, the objective functions are essentially the same; we maximize the sum of the agents' utilities/profits over possible matchings. The constraint functions vary across problems and are dealt with separately in order to guarantee the hierarchical structure.

Let $N=D \cup H$. Consider the domain $\{-1,0,1\}^{N \times \Omega}$. For $x \in\{-1,0,1\}^{N \times \Omega}$, $x_{(d,(d, h))}=1$ (or -1$)$ is intended to mean that $d$ is matched to $h$. Fig. 3 visualizes the constraints in each problem.

In the first maximization problem, we set the constraint functions to represent the doctors' capacity constraints, i.e., each doctor is matched to at most one hospital (see Fig. 3 (a)). We require that the sum of the values in each shaded area be no greater than 0 . If the sum is equal to 0 , then we can realize a matching; $d$ is matched to $h$ if the value of $(d,(d, h))$ is -1 , which entails that the value of $(h,(d, h))$ is 1 and $h$ is matched to $d$. It is possible that the sum is equal to -1 , but this case can be circumvented at optimal solutions by utilizing the complementary slackness and monotonicity of revenue functions (see the argument after (48) in the formal proof).

The second problem deals with the floor constraints (see Fig. 3 (b)), and the third deals with the ceiling constraints (see Fig. 3 (c)). These two can be handled analogously by exchanging -1 and +1 denoting the matching structure.

It is clear from Fig. 3 that, in each independent problem, the constraint structures form a hierarchy (they are mutually disjoint), and hence, Theorem 1 can be applied. We translate a saddle point into the min-max form of the Lagrange function. This transformation enables us to aggregate the results in each maximization problem into a single function.

\subsubsection{Preliminaries for the proof of $(i) \Rightarrow$ (ii)}

Let $s \in \mathbb{Z}_{+}^{\Omega}$. For each $d \in D$, we define $\tilde{v}_{d}[s]: \mathbb{Z}^{\Omega} \rightarrow \mathbb{Z} \cup\{-\infty\}$ by

$$
\tilde{v}_{d}[s](x)= \begin{cases}v_{d}[s](h) & \text { if } x \in\{0,1\}^{\Omega} \text { and there exists } h \in H \text { such that } \\ & x_{(d, h)}=1, x_{\left(d, h^{\prime}\right)}=0 \text { for all } h^{\prime} \neq h, \text { and } \\ & x_{\left(d^{\prime}, h^{\prime \prime}\right)}=0 \text { for all } d^{\prime} \neq d \text { and } h^{\prime \prime} \in H, \\ v_{d}[s]\left(h_{0}\right) & \text { if } x=\mathbf{0}, \\ -\infty & \text { otherwise }\end{cases}
$$




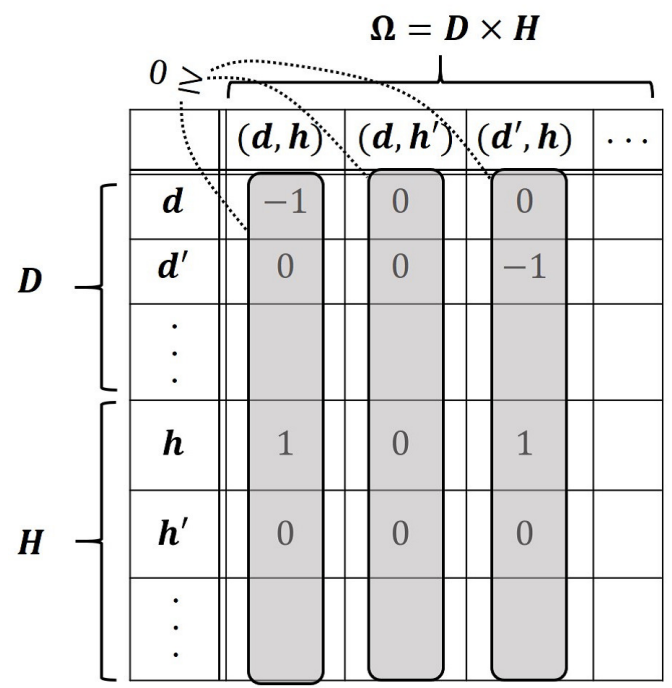

(a)

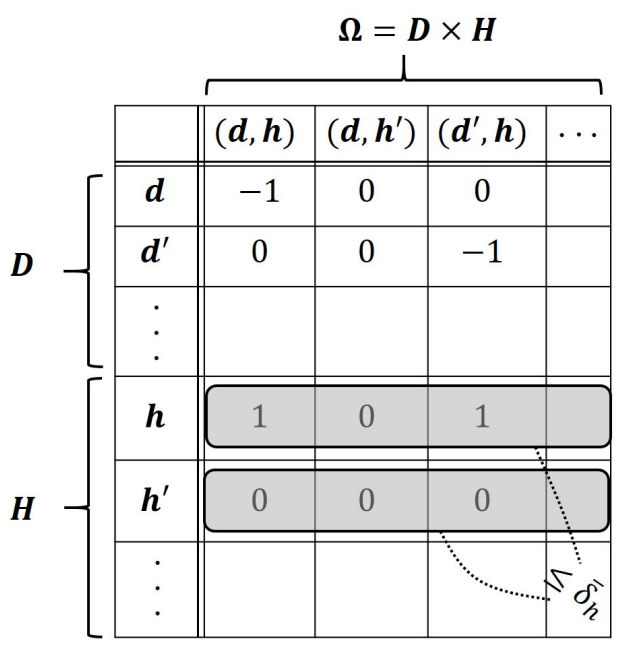

(b)

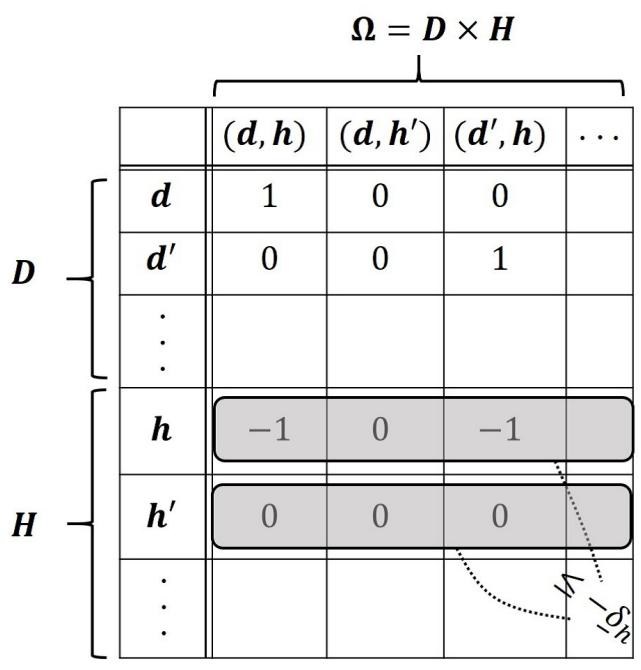

(c)

Fig. 3 Constraints in each of the three maximization problems. 
Since the effective domain of $\tilde{v}_{d}[s](\cdot)$ consists of unit vectors, $\tilde{v}_{d}[s](\cdot)$ is $\mathrm{M}^{\natural}$-concave.

Let $s \in \mathbb{Z}_{+}^{\Omega}$ and $t \in \mathbb{Z}^{H}$. For each $h \in H$, we define $\tilde{v}_{h}[s, t]: \mathbb{Z}^{\Omega} \rightarrow$ $\mathbb{Z} \cup\{-\infty\}$ by

$$
\tilde{v}_{h}[s, t](x)= \begin{cases}v_{h}[s, t]\left(\left\{d \in D: x_{(d, h)}=1\right\}\right) & \text { if } x \in\{0,1\}^{\Omega} \text { and } \\ & x_{\left(d, h^{\prime}\right)}=0 \text { for all } d \in D \text { and } h^{\prime} \neq h \\ -\infty & \text { otherwise }\end{cases}
$$

By Proposition $1, v_{h}(\cdot)$ is $\mathrm{M}^{\natural}$-concave. By Theorem 6.15 of Murota (2003a), $v_{h}[s, t](\cdot)$ is $\mathbf{M}^{\natural}$-concave. Hence, $\tilde{v}_{h}[s, t](\cdot)$ is also $\mathbf{M}^{\natural}$-concave.

For $x \in\{-1,0,1\}^{N \times \Omega}$ and $i \in N$, let $\left.x\right|_{i}$ denote the projection of $x$ on $\{-1,0,1\}^{\{i\} \times \Omega}$; we often identify $\{-1,0,1\}^{\{i\} \times \Omega}$ with $\{-1,0,1\}^{\Omega}$. For $x \in\{-1,0,1\}^{N \times \Omega}$, let $x_{D}$ and $x_{H}$ denote the projections of $x$ on $\{-1,0,1\}^{D \times \Omega}$ and $\{-1,0,1\}^{H \times \Omega}$, respectively.

We formally describe the three maximization problems discussed in Section 5.3.2.

Maximization problem 1: We fix $\underline{t} \in \mathbb{Z}_{+}^{H}$ and $\bar{t} \in \mathbb{Z}_{+}^{H}$ throughout this problem. We define $f^{1}: \mathbb{Z}^{N \times \Omega} \rightarrow \mathbb{Z} \cup\{-\infty\}$ by

$$
f^{1}(x)=\sum_{d \in D} \tilde{v}_{d}[\mathbf{0}]\left(-\left.x\right|_{d}\right)+\sum_{h \in H} \tilde{v}_{h}[\mathbf{0}, \underline{t}-\bar{t}]\left(\left.x\right|_{h}\right)+G(-\bar{t})+G(\underline{t}) \text { for all } x \in \mathbb{Z}^{N \times \Omega} .
$$

One can verify that, if $\tilde{v}_{d}[\mathbf{0}](\cdot)$ and $\tilde{v}_{h}[\mathbf{0}, \underline{t}-\bar{t}](\cdot)$ are $\mathbf{M}^{\natural}$-concave, then $f^{1}(\cdot)$ is also $\mathbf{M}^{\natural}$-concave. ${ }^{21}$ Note that $\operatorname{dom} f^{1} \subseteq\{-1,0,1\}^{N \times \Omega}$.

For each $\omega \in \Omega$, we define

$$
g_{\omega}(x)=-x \cdot \mathbb{1}^{N \times\{\omega\}} \text { for all } x \in \mathbb{Z}^{N \times \Omega} .
$$

We consider the following problem:

$$
\max _{x \in\{-1,0,1\}^{N \times \Omega}} f^{1}(x) \text { subject to } g_{\omega}(x) \geq 0 \text { for all } \omega \in \Omega .
$$

${ }^{21}$ Fujishige and Tamura (2007) utilize this preservation of $\mathrm{M}^{\natural}$-concavity in the analysis of two-sided markets (see equation (15) therein). 
Let $x^{*}$ be a solution to (M1). Then, by Theorem 1 , there exists $s^{*} \in \mathbb{Z}_{+}^{\Omega}$ such that $^{22}$

$$
\begin{aligned}
& L\left(x^{*}, s^{*}\right) \\
& =\min _{s \in \mathbb{Z}_{+}^{\Omega}} \max _{x \in\{-1,0,1\}^{N \times \Omega}} L(x, s) \\
& =\min _{s \in \mathbb{Z}_{+}^{\Omega}} \max _{x \in\{-1,0,1\}^{N \times \Omega}}\left\{\sum_{d \in D} \tilde{v}_{d}[\mathbf{0}]\left(-\left.x\right|_{d}\right)+\sum_{h \in H} \tilde{v}_{h}[\mathbf{0}, \underline{t}-\bar{t}]\left(\left.x\right|_{h}\right)\right. \\
& \left.+G(-\bar{t})+G(\underline{t})+\sum_{\omega \in \Omega} s_{\omega} g_{\omega}(x)\right\} \\
& =\min _{s \in \mathbb{Z}_{+}^{\Omega}} \max _{x \in\{-1,0,1\}^{N \times \Omega}}\left\{\sum_{d \in D} \tilde{v}_{d}[\mathbf{0}]\left(-\left.x\right|_{d}\right)+\sum_{h \in H} \tilde{v}_{h}[\mathbf{0}, \underline{t}-\bar{t}]\left(\left.x\right|_{h}\right)\right. \\
& \left.+G(-\bar{t})+G(\underline{t})-\sum_{\omega \in \Omega} s_{\omega} \sum_{i \in N}\left(\left.x\right|_{i}\right)_{\omega}\right\} \\
& =\min _{s \in \mathbb{Z}_{+}^{\Omega}} \max _{x \in\{-1,0,1\}^{N \times \Omega}}\left\{\sum_{d \in D}\left(\tilde{v}_{d}[\mathbf{0}]\left(-\left.x\right|_{d}\right)-\left.s \cdot x\right|_{d}\right)+\sum_{h \in H}\left(\tilde{v}_{h}[\mathbf{0}, \underline{t}-\bar{t}]\left(\left.x\right|_{h}\right)-\left.s \cdot x\right|_{h}\right)\right. \\
& +G(-\bar{t})+G(\underline{t})\} \\
& =\min _{s \in \mathbb{Z}_{+}^{\Omega}} \max _{x \in\{0,1\}^{N \times \Omega}}\left\{\sum_{d \in D} \tilde{v}_{d}[s]\left(\left.x\right|_{d}\right)+\sum_{h \in H} \tilde{v}_{h}[s, \underline{t}-\bar{t}]\left(\left.x\right|_{h}\right)+G(-\bar{t})+G(\underline{t})\right\} .
\end{aligned}
$$

Note that, to derive the last equality, we change the domain for choosing $\left.x\right|_{d}$ from $\{0,-1\}^{\{d\} \times \Omega}$ to $\{0,1\}^{\{d\} \times \Omega}$. Together with the complementary slackness, we obtain the following claim:

Claim 7. Let $\underline{t} \in \mathbb{Z}_{+}^{H}$ and $\bar{t} \in \mathbb{Z}_{+}^{H}$. Then, for $x^{*} \in\{0,1\}^{N \times \Omega}$, the following statements are equivalent:

- $\left(-x_{D}^{*}, x_{H}^{*}\right)$ is a solution to $(M 1)$.

- There exists $s^{*} \in \mathbb{Z}_{+}^{\Omega}$ such that $\left(x^{*}, s^{*}\right)$ is a solution to

$$
\min _{s \in \mathbb{Z}_{+}^{\Omega}} \max _{x \in\{0,1\}^{N \times \Omega}}\left\{\sum_{d \in D} \tilde{v}_{d}[s]\left(\left.x\right|_{d}\right)+\sum_{h \in H} \tilde{v}_{h}[s, \underline{t}-\bar{t}]\left(\left.x\right|_{h}\right)+G(-\bar{t})+G(\underline{t})\right\} .
$$

Moreover, ${ }^{*}$ satisfies

$$
s_{\omega}^{*} \cdot g_{\omega}\left(x^{*}\right)=0 \text { for all } \omega \in \Omega .
$$

${ }^{22}$ Below the first equality follows because $L\left(x^{*}, s^{*}\right)=\min _{s} L\left(x^{*}, s\right) \leq \max _{x} \min _{s} L(x, s) \leq$ $\min _{s} \max _{x} L(x, s) \leq \max _{x} L\left(x, s^{*}\right)=L\left(x^{*}, s^{*}\right)$. 
Maximization problem 2: We fix $s \in \mathbb{Z}_{+}^{\Omega}$ and $\underline{t} \in \mathbb{Z}_{+}^{H}$ throughout this problem. We define $f^{2}: \mathbb{Z}^{N \times \Omega} \rightarrow \mathbb{Z} \cup\{-\infty\}$ by

$$
f^{2}(x)=\sum_{d \in D} \tilde{v}_{d}[s]\left(\left.x\right|_{d}\right)+\sum_{h \in H} \tilde{v}_{h}[s, \underline{t}]\left(\left.x\right|_{h}\right)+G(\underline{t}) \text { for all } x \in \mathbb{Z}^{N \times \Omega} .
$$

For each $h \in H$, we define

$$
\bar{g}_{h}(x)=\bar{\delta}_{h}-x \cdot \mathbb{1}^{\{h\} \times \Omega} \text { for all } x \in \mathbb{Z}^{N \times \Omega} .
$$

We consider the following problem:

$$
\max _{x \in\{0,1\}^{\Omega}} f^{2}(x) \text { subject to } \bar{g}_{h}(x) \geq 0 \text { for all } h \in H .
$$

Let $x^{*}$ be a solution to (M2). Then, by Theorem 1, there exists $\bar{t}^{*}$ such that

$$
\begin{aligned}
& L\left(x^{*}, \bar{t}^{*}\right) \\
& =\min _{\bar{t} \in \mathbb{Z}_{+}^{H}} \max _{x \in\{0,1\}^{N \times \Omega}} L(x, \bar{t}) \\
& =\min _{\bar{t} \in \mathbb{Z}_{+}^{H}} \max _{x \in\{0,1\}^{N \times \Omega}}\left\{\sum_{d \in D} \tilde{v}_{d}[s]\left(\left.x\right|_{d}\right)+\sum_{h \in H} \tilde{v}_{h}[s, \underline{t}]\left(\left.x\right|_{h}\right)+G(\underline{t})+\sum_{h \in H} \bar{t}_{h} \bar{g}_{h}(x)\right\} \\
& =\min _{\bar{t} \in \mathbb{Z}_{+}^{H}} \max _{x \in\{0,1\}^{N \times \Omega}}\left\{\sum_{d \in D} \tilde{v}_{d}[s]\left(\left.x\right|_{d}\right)+\sum_{h \in H}\left(\tilde{v}_{h}[s, \underline{t}]\left(\left.x\right|_{h}\right)-\bar{t}_{h} \cdot x \cdot \mathbb{1}^{\{h\} \times \Omega}\right)\right. \\
& \left.+G(\underline{t})+\sum_{h \in H} \bar{\delta}_{h} \cdot \bar{t}_{h}\right\} \\
& =\min _{\bar{t} \in \mathbb{Z}_{+}^{H}} \max _{x \in\{0,1\}^{N \times \Omega}}\left\{\sum_{d \in D} \tilde{v}_{d}[s]\left(\left.x\right|_{d}\right)+\sum_{h \in H} \tilde{v}_{h}[s, \underline{t}-\bar{t}]\left(\left.x\right|_{h}\right)+G(\underline{t})+G(-\bar{t})\right\} .
\end{aligned}
$$

Together with the complementary slackness, we obtain the following claim:

Claim 8. Let $s \in \mathbb{Z}_{+}^{\Omega}$ and $\underline{t} \in \mathbb{Z}_{+}^{H}$. Then, for $x^{*} \in\{0,1\}^{N \times \Omega}$, the following statements are equivalent:

- $x^{*}$ is a solution to (M2).

- There exists $\bar{t}^{*}$ such that $\left(x^{*}, \bar{t}^{*}\right)$ is a solution to

$$
\min _{\bar{t} \in \mathbb{Z}_{+}^{H}} \max _{x \in\{0,1\}^{N \times \Omega}}\left\{\sum_{d \in D} \tilde{v}_{d}[s]\left(\left.x\right|_{d}\right)+\sum_{h \in H} \tilde{v}_{h}[s, \underline{t}-\bar{t}]\left(\left.x\right|_{h}\right)+G(\underline{t})+G(-\bar{t})\right\} .
$$

Moreover, $\bar{t}^{*}$ satisfies

$$
\bar{t}_{h}^{*} \cdot \bar{g}_{h}\left(x^{*}\right)=0 \text { for all } h \in H .
$$


Maximization problem 3: We fix $s \in \mathbb{Z}_{+}^{\Omega}$ and $\bar{t} \in \mathbb{Z}_{+}^{H}$ throughout this problem. We define $f^{3}: \mathbb{Z}^{N \times \Omega} \rightarrow \mathbb{Z} \cup\{-\infty\}$ by ${ }^{23}$

$$
f^{3}(x)=\sum_{d \in D} \tilde{v}_{d}[s]\left(\left.x\right|_{d}\right)+\sum_{h \in H} \tilde{v}_{h}[s,-\bar{t}]\left(-\left.x\right|_{h}\right)+G(-\bar{t}) \text { for all } x \in \mathbb{Z}^{N \times \Omega} .
$$

For each $h \in H$, we define

$$
\underline{g}_{h}(x)=-\underline{\delta}_{h}-x \cdot \mathbb{1}^{\{h\} \times \Omega} \text { for all } x \in \mathbb{Z}^{N \times \Omega} .
$$

We consider the following problem:

$$
\max _{x \in\{-1,0,1\}^{N \times \Omega}} f^{3}(x) \text { subject to } \underline{g}_{h}(x) \geq 0 \text { for all } h \in H .
$$

Let $x^{*}$ be a solution to (M3). Then, by Theorem 1 , there exists $\underline{t}^{*}$ such that

$$
\begin{aligned}
& L\left(x^{*}, \underline{t}^{*}\right) \\
& =\min _{\underline{t} \in \mathbb{Z}_{+}^{H}} \max _{x \in\{-1,0,1\}^{N \times \Omega}} L(x, \underline{t}) \\
& =\min _{\underline{t} \in \mathbb{Z}_{+}^{H}} \max _{x \in\{-1,0,1\}^{N \times \Omega}}\left\{\sum_{d \in D} \tilde{v}_{d}[s]\left(\left.x\right|_{d}\right)+\sum_{h \in H} \tilde{v}_{h}[s,-\bar{t}]\left(-\left.x\right|_{h}\right)\right. \\
& \left.+G(-\bar{t})+\sum_{h \in H} \underline{t}_{h} \underline{g}_{h}(x)\right\} \\
& =\min _{\underline{t} \in \mathbb{Z}_{+}^{H}} \max _{x \in\{-1,0,1\}^{N \times \Omega}}\left\{\sum_{d \in D} \tilde{v}_{d}[s]\left(\left.x\right|_{d}\right)+\sum_{h \in H}\left(\tilde{v}_{h}[s,-\bar{t}]\left(-\left.x\right|_{h}\right)-\underline{t}_{h} \cdot x \cdot \mathbb{1}^{\{h\} \times \Omega}\right)\right. \\
& \left.+G(-\bar{t})-\sum_{h \in H} \underline{\delta}_{h} \cdot \underline{t}_{h}\right\} \\
& =\min _{\underline{t} \in \mathbb{Z}_{+}^{H}} \max _{x \in\{0,1\}^{N \times \Omega}}\left\{\sum_{d \in D} \tilde{v}_{d}[s]\left(\left.x\right|_{d}\right)+\sum_{h \in H} \tilde{v}_{h}[s, \underline{t}-\bar{t}]\left(\left.x\right|_{h}\right)+G(-\bar{t})+G(\underline{t})\right\} .
\end{aligned}
$$

Note that, to derive the last equality, we change the domain for choosing $\left.x\right|_{h}$ from $\{0,-1\}^{\{h\} \times \Omega}$ to $\{0,1\}^{\{h\} \times \Omega}$. Together with the complementary slackness, we obtain the following claim:

Claim 9. Let $s \in \mathbb{Z}_{+}^{\Omega}$ and $\bar{t} \in \mathbb{Z}_{+}^{H}$. Then, for $x^{*} \in\{0,1\}^{N \times \Omega}$, the following statements are equivalent:

${ }^{23}$ It is known that if a function $f(x)$ is $\mathrm{M}^{\natural}$-concave in $x$, then $f(-x)$ is also $\mathrm{M}^{\natural}$-concave in $x$; see Theorem 6.15 of Murota (2003a). Hence, $f^{3}(x)$ is $\mathrm{M}^{\natural}$-concave in $x$. 
- $\left(x_{D}^{*},-x_{H}^{*}\right)$ is a solution to (M3).

- There exists $\underline{t}^{*} \in \mathbb{Z}_{+}^{H}$ such that $\left(x^{*}, \underline{t}^{*}\right)$ is a solution to

$$
\min _{\underline{t} \in \mathbb{Z}_{+}^{H}} \max _{x \in\{0,1\}^{N \times \Omega}}\left\{\sum_{d \in D} \tilde{v}_{d}[s]\left(\left.x\right|_{d}\right)+\sum_{h \in H} \tilde{v}_{h}[s, \underline{t}-\bar{t}]\left(\left.x\right|_{h}\right)+G(-\bar{t})+G(\underline{t})\right\} .
$$

Moreover, $\underline{t}^{*}$ satisfies

$$
\underline{t}_{h}^{*} \cdot \underline{g}_{h}\left(x^{*}\right)=0 \text { for all } h \in H .
$$

\subsubsection{Proof of $(i) \Rightarrow$ (ii)}

We define $\hat{F}: \mathbb{Z}^{H} \times \mathbb{Z}_{+}^{\Omega} \times \mathbb{Z}^{N \times \Omega} \rightarrow \mathbb{Z} \cup\{-\infty\}$ and $\check{F}: \mathbb{Z}_{+}^{H} \times \mathbb{Z}_{+}^{H} \times \mathbb{Z}_{+}^{\Omega} \times \mathbb{Z}^{N \times \Omega} \rightarrow$ $\mathbb{Z} \cup\{-\infty\}$ by

$$
\begin{aligned}
\hat{F}(t, s, x)= & \sum_{d \in D} \tilde{v}_{d}[s]\left(\left.x\right|_{d}\right)+\sum_{h \in H} \tilde{v}_{h}[s, t]\left(\left.x\right|_{h}\right)+G(t) \\
& \text { for all }(t, s, x) \in \mathbb{Z}^{H} \times \mathbb{Z}_{+}^{\Omega} \times \mathbb{Z}^{N \times \Omega}, \\
\check{F}(\bar{t}, \underline{t}, s, x)= & \sum_{d \in D} \tilde{v}_{d}[s]\left(\left.x\right|_{d}\right)+\sum_{h \in H} \tilde{v}_{h}[s, \underline{t}-\bar{t}]\left(\left.x\right|_{h}\right)+G(-\bar{t})+G(\underline{t}) \\
& \text { for all }(\bar{t}, \underline{t}, s, x) \in \mathbb{Z}_{+}^{H} \times \mathbb{Z}_{+}^{H} \times \mathbb{Z}_{+}^{\Omega} \times \mathbb{Z}^{N \times \Omega} .
\end{aligned}
$$

We prove two claims.

Claim 10. Suppose that there exists a solution to

$$
\min _{t \in \mathbb{Z}^{H}} \min _{s \in \mathbb{Z}^{\Omega}} \max _{x \in\{0,1\}^{N \times \Omega}} \hat{F}(t, s, x) .
$$

Then, there also exists a solution to

$$
\min _{\bar{t} \in \mathbb{Z}_{+}^{H}} \min _{t \in \mathbb{Z}_{+}^{H}} \min _{s \in \mathbb{Z}^{\Omega}} \max _{x \in\{0,1\}^{N \times \Omega}} \check{F}(\bar{t}, \underline{t}, s, x),
$$

and $(32)=(33)$. In particular, for a solution $\left(t^{*}, s^{*}, x^{*}\right)$ to (32), there exist $\bar{t}^{*} \in \mathbb{Z}_{+}^{H}$ and $\underline{t}^{*} \in \mathbb{Z}_{+}^{H}$ such that $\underline{t}^{*}-\bar{t}^{*}=t^{*}$ and $\left(\bar{t}^{*}, \underline{t}^{*}, s^{*}, x^{*}\right)$ is a solution to (33). 
Proof. Proof of (32) $\leq$ (33). Let $(\bar{t}, \underline{t}, s) \in \mathbb{Z}_{+}^{H} \times \mathbb{Z}_{+}^{H} \times \mathbb{Z}_{+}^{\Omega}$ be arbitrarily chosen. It suffices to prove that there exists $t \in \mathbb{Z}^{H}$ such that

$$
\max _{x \in\{0,1\}^{N \times \Omega}} \hat{F}(t, s, x) \leq \max _{x \in\{0,1\}^{N \times \Omega}} \check{F}(\bar{t}, \underline{t}, s, x) .
$$

Suppose that there exists $h^{\prime} \in H$ with $\bar{t}_{h^{\prime}}>0$ and $\underline{t}_{h^{\prime}}>0$. We define $\bar{t}^{*} \in \mathbb{Z}_{+}^{H}$ and $t^{*} \in \mathbb{Z}_{+}^{H}$ as follows:

$$
\bar{t}_{h^{\prime}}^{*}=\bar{t}_{h^{\prime}}-1, \underline{t}_{h^{\prime}}^{*}=\underline{t}_{h^{\prime}}-1, \bar{t}^{*}=\left(\left(\bar{t}_{h}\right)_{h \neq h^{\prime}}, \bar{t}_{h^{\prime}}^{*}\right), \underline{t}^{*}=\left(\left(\underline{t}_{h}\right)_{h \neq h^{\prime}}, \underline{t}_{h^{\prime}}^{*}\right) .
$$

Then,

$$
G\left(-\bar{t}^{*}\right)+G\left(\underline{t}^{*}\right)=\left\{G(-\bar{t})-\bar{\delta}_{h^{\prime}}\right\}+\left\{G(\underline{t})+\underline{\delta}_{h^{\prime}}\right\} \leq G(-\bar{t})+G(\underline{t}),
$$

where the inequality follows from $\underline{\delta}_{h^{\prime}} \leq \bar{\delta}_{h^{\prime}}$. Moreover, since $\underline{t}-\bar{t}=\underline{t}^{*}-\bar{t}^{*}$, we obtain

$$
\check{F}\left(\bar{t}^{*}, \underline{t}^{*}, s, x\right) \leq \check{F}(\bar{t}, \underline{t}, s, x) \text { for all } x \in\{0,1\}^{N \times \Omega} .
$$

This inequality implies

$$
\max _{x \in\{0,1\}^{N \times \Omega}} \check{F}\left(\bar{t}^{*}, \underline{t}^{*}, s, x\right) \leq \max _{x \in\{0,1\}^{N \times \Omega}} \check{F}(\bar{t}, \underline{t}, s, x) .
$$

By repeating the above procedure for all $h \in H$ with $\bar{t}_{h}>0$ and $\underline{t}_{h}>0$, we can construct $\bar{t}^{* *} \in \mathbb{Z}_{+}^{H}$ and $\underline{t}^{* *} \in \mathbb{Z}_{+}^{H}$ such that

$$
\begin{aligned}
& \max _{x \in\{0,1\}^{N \times \Omega}} \check{F}\left(\bar{t}^{* *}, \underline{t}^{* *}, s, x\right) \leq \max _{x \in\{0,1\}^{N \times \Omega}} \check{F}(\bar{t}, \underline{t}, s, x), \text { and } \\
& \text { for any } h \in H, \bar{t}_{h}^{* *}=0 \text { or } \underline{t}_{h}^{* *}=0 \text { holds. }
\end{aligned}
$$

By (36), for any $h \in H$,

$$
G_{h}\left(-\bar{t}_{h}^{* *}\right)+G_{h}\left(\underline{t}_{h}^{* *}\right)= \begin{cases}G_{h}\left(-\bar{t}_{h}^{* *}\right)+0 & \text { if } \bar{t}_{h}^{* *}>0 \\ 0+G_{h}\left(\underline{t}_{h}^{* *}\right) & \text { if } \bar{t}_{h}^{* *}=0\end{cases}
$$

We define $t \in \mathbb{Z}^{H}$ as follows: for each $h \in H$,

$$
t_{h}= \begin{cases}-\bar{t}_{h}^{* *} & \text { if } \bar{t}_{h}^{* *}>0 \\ \underline{t}_{h}^{* *} & \text { if } \bar{t}_{h}^{* *}=0\end{cases}
$$


Then, (37) and (38) imply that $G_{h}\left(-\bar{t}_{h}^{* *}\right)+G_{h}\left(\underline{t}_{h}^{* *}\right)=G_{h}\left(t_{h}\right)$. Taking the sum over $h \in H$, we obtain $G\left(-\bar{t}^{* *}\right)+G\left(\underline{t}^{* *}\right)=G(t)$. Moreover, by (38) and (36), for any $h \in H$,

$$
\begin{aligned}
t_{h} & = \begin{cases}0-\bar{t}_{h}^{* *} & \text { if } \bar{t}_{h}^{* *}>0, \\
\underline{t}_{h}^{* *}-0 & \text { if } \bar{t}_{h}^{* *}=0,\end{cases} \\
& = \begin{cases}\underline{t}_{h}^{* *}-\bar{t}_{h}^{* *} & \text { if } \bar{t}_{h}^{* *}>0, \\
\underline{t}_{h}^{* *}-\bar{t}_{h}^{* *} & \text { if } \bar{t}_{h}^{* *}=0,\end{cases} \\
& =\underline{t}_{h}^{* *}-\bar{t}_{h}^{* *} .
\end{aligned}
$$

It follows that $t=\underline{t}^{* *}-\bar{t}^{* *}$. Together with $G\left(-\bar{t}^{* *}\right)+G\left(\underline{t}^{* *}\right)=G(t)$,

$$
\hat{F}(t, s, x)=\check{F}\left(\underline{t}^{* *}, \bar{t}^{* *}, s, x\right) \text { for all } x \in\{0,1\}^{N \times \Omega} \text {. }
$$

This equation implies

$$
\max _{x \in\{0,1\}^{N \times \Omega}} \hat{F}(t, s, x)=\max _{x \in\{0,1\}^{N \times \Omega}} \check{F}\left(\underline{t}^{* *}, \bar{t}^{* *}, s, x\right) .
$$

Together with (35), we obtain (34).

Proof of (32) $\geq(33)$ : Let $\left(t^{*}, s^{*}, x^{*}\right)$ be a solution to (32). We define $\bar{t}^{*}=\left(\bar{t}_{h}^{*}\right)_{h \in H}$ and $\underline{t}^{*}=\left(\underline{t}_{h}^{*}\right)_{h \in H}$ as follows: for each $h \in H$,

$$
\bar{t}_{h}^{*}=\left\{\begin{array}{ll}
-t_{h}^{*} & \text { if } t_{h}^{*} \leq 0, \\
0 & \text { if } t_{h}^{*}>0,
\end{array} \quad t_{h}^{*}= \begin{cases}0 & \text { if } t_{h}^{*} \leq 0 \\
t_{h}^{*} & \text { if } t_{h}^{*}>0\end{cases}\right.
$$

Then, for any $h \in H$,

$$
G_{h}\left(-\bar{t}_{h}^{*}\right)=\left\{\begin{array}{ll}
G_{h}\left(t_{h}^{*}\right) & \text { if } t_{h}^{*} \leq 0, \\
0 & \text { if } t_{h}^{*}>0,
\end{array} G_{h}\left(\underline{t}_{h}^{*}\right)= \begin{cases}0 & \text { if } t_{h}^{*} \leq 0 \\
G_{h}\left(t_{h}^{*}\right) & \text { if } t_{h}^{*}>0 .\end{cases}\right.
$$

These equations imply $G_{h}\left(-\bar{t}_{h}^{*}\right)+G_{h}\left(t_{h}^{*}\right)=G_{h}\left(t_{h}^{*}\right)$. Taking the sum over $h \in H$, we obtain $G\left(-\bar{t}^{*}\right)+G\left(\underline{t}^{*}\right)=G\left(t^{*}\right)$. Moreover, since $\underline{t}^{*}-\bar{t}^{*}=t^{*}$ by (39),

$$
\hat{F}\left(t^{*}, s^{*}, x\right)=\check{F}\left(\bar{t}^{*}, \underline{t}^{*}, s^{*}, x\right) \text { for all } x \in\{0,1\}^{N \times \Omega} .
$$


This equation implies

$$
\text { (32) }=\hat{F}\left(t^{*}, s^{*}, x^{*}\right)=\max _{x \in\{0,1\}^{N \times \Omega}} \hat{F}\left(t^{*}, s^{*}, x\right)=\max _{x \in\{0,1\}^{N \times \Omega}} \check{F}\left(\bar{t}^{*}, \underline{t}^{*}, s^{*}, x\right) \geq(33),
$$

where the inequality holds because (33) takes the minimum value over $(\bar{t}, \underline{t}, s) \in$ $\mathbb{Z}_{+}^{H} \times \mathbb{Z}_{+}^{H} \times \mathbb{Z}_{+}^{\Omega}$.

As proven in the first half of the proof, (33) $\geq(32)$. Together with the above inequality, $(33)=(32)$. Moreover, by $(40),\left(\bar{t}^{*}, t^{*}, s^{*}, x^{*}\right)$ is a solution to (33). Finally, as mentioned above, (39) implies $\underline{t}^{*}-\bar{t}^{*}=t^{*}$ and hence the last part of the statement follows.

Claim 11. Let $(s, t) \in \mathbb{Z}_{+}^{\Omega} \times \mathbb{Z}^{H}$. Then,

$$
\max _{x \in\{0,1\}^{N \times \Omega}} \hat{F}(t, s, x)=F(s, t) \equiv \sum_{d \in D} V_{d}(s)+\sum_{h \in H} V_{h}(s, t)+G(t) .
$$

Proof. This equation immediately follows from the definition of indirect utility functions.

We start the proof of (i) $\Rightarrow$ (ii). Let $\left(s^{*}, t^{*}\right)$ be a solution to (8). Let $x^{*}$ be a solution to

$$
\max _{x \in\{0,1\}^{N \times \Omega}} \hat{F}\left(t^{*}, s^{*}, x\right) .
$$

Then,

$$
\begin{aligned}
\hat{F}\left(t^{*}, s^{*}, x^{*}\right) & =\max _{x \in\{0,1\}^{N \times \Omega}} \hat{F}\left(t^{*}, s^{*}, x\right) \\
& =F\left(s^{*}, t^{*}\right) \\
& =\min _{t \in \mathbb{Z}^{H}} \min _{s \in \mathbb{Z}_{+}^{\Omega}} F(s, t) \\
& =\min _{t \in \mathbb{Z}^{H}} \min _{s \in \mathbb{Z}_{+}^{\Omega}} \max _{x \in\{0,1\}^{N \times \Omega}} \hat{F}(t, s, x),
\end{aligned}
$$

where the scond and fourth equalities follow from Claim 11. It follows that $\left(t^{*}, s^{*}, x^{*}\right)$ is a solution to (32). By Claim 10, setting $\bar{t}^{*}$ and $\underline{t}^{*}$ as in (39), $\left(\bar{t}^{*}, \underline{t}^{*}, s^{*}, x^{*}\right)$ is a solution to (33) and satisfies

$$
\underline{t}^{*}-\bar{t}^{*}=t^{*}
$$


Then, ${ }^{24}$

$$
\begin{aligned}
& \check{F}\left(\bar{t}^{*}, \underline{t}^{*}, s^{*}, x^{*}\right) \\
= & \min _{\bar{t} \in \mathbb{Z}_{+}^{H}} \min _{t \in \mathbb{Z}_{+}^{H}} \min _{s \in \mathbb{Z}_{+}^{\Omega}} \max _{x \in\{0,1\}^{N \times \Omega}} \check{F}(\bar{t}, \underline{t}, s, x) \\
= & \min _{s \in \mathbb{Z}_{+}^{\Omega}} \max _{x \in\{0,1\}^{N \times \Omega}} \check{F}\left(\bar{t}^{*}, \underline{t}^{*}, s, x\right) \\
= & \min _{\bar{t} \in \mathbb{Z}_{+}^{H}} \max _{x \in\{0,1\}^{N \times \Omega}} \check{F}\left(\bar{t}, \underline{t}^{*}, s^{*}, x\right) \\
= & \min _{\underline{t} \in \mathbb{Z}_{+}^{H}} \max _{x \in\{0,1\}^{N \times \Omega}} \check{F}\left(\bar{t}^{*}, \underline{t}, s^{*}, x\right) \\
= & \max _{x \in\{0,1\}^{N \times \Omega}} \check{F}\left(\bar{t}^{*}, \underline{t}^{*}, s^{*}, x\right) .
\end{aligned}
$$

By $(43)=(45)$ and Claim $7,\left(-x_{D}^{*}, x_{H}^{*}\right)$ is a solution to (M1). Suppose that $g_{\omega}\left(-x_{D}^{*}, x_{H}^{*}\right)>0$ for some $\omega \in \Omega$ and let $\left(d^{\prime}, h^{\prime}\right) \equiv \omega$. Then, $x_{\left(d^{\prime},\left(d^{\prime}, h^{\prime}\right)\right)}^{*}=1$ and $x_{\left(h^{\prime},\left(d^{\prime}, h^{\prime}\right)\right)}^{*}=0$. By (29), $s_{\left(d^{\prime}, h^{\prime}\right)}^{*}=0$. Together with monotonicity of $v_{h^{\prime}}(\cdot)$, replacing the $\left(h^{\prime},\left(d^{\prime}, h^{\prime}\right)\right)$-th coordinate of $x^{*}$ with 1 yields a solution to (41). Hence, w.l.o.g., we assume that $\left(-x_{D}^{*}, x_{H}^{*}\right)$ is a solution to (M1) satisfying all the constraints with equality. Namely, for each $\omega \in \Omega$,

$$
0=g_{\omega}\left(-x_{D}^{*}, x_{H}^{*}\right)=\left(-x_{D}^{*}, x_{H}^{*}\right) \cdot \mathbb{1}^{N \times\{\omega\}}=-\sum_{d \in D}\left(\left.x^{*}\right|_{d}\right)_{\omega}+\sum_{h \in H}\left(\left.x^{*}\right|_{h}\right)_{\omega} .
$$

Then, defining $\mu: D \rightarrow H$ by

$$
\mu(d)=h \Longleftrightarrow x_{(d,(d, h))}^{*}=1 \text { for all } d \in D \text { and } h \in H,
$$

$\mu$ forms a matching.

By $(43)=(46)$ and Claim $8, x^{*}$ is a solution to (M2). Hence, $x^{*}$ satisfies the ceiling constraints. By $(43)=(47)$ and Claim $9,\left(x_{D}^{*},-x_{H}^{*}\right)$ is a solution to (M3). Hence, $x^{*}$ satisfies the floor constraints. It follows that $\mu$ is feasible. Moreover, by (42) and (43)=(48), $x^{*}$ maximizes every agent's utilities/profits under the salary system $s^{*}$ and the transfer system $t^{*}$. We conclude that $\left(\mu, s^{*}, t^{*}\right)$ forms an uncompelled competitive equilibrium.

${ }^{24}$ In the transformation below, one can verify the following equations: $(44)=(45),(44)=(46)$, $(44)=(47)$, and $(44)=(48)$. 
It remains to prove that (5) holds. If $t_{h}^{*}<0$, since $\bar{t}_{h}^{*}$ is constructed by (39), we obtain $\bar{t}_{h}^{*}>0$. By (30), for any $h \in H$,

$$
\begin{aligned}
\bar{g}_{h}\left(x^{*}\right) & =\bar{\delta}_{h}-x^{*} \cdot \mathbb{1}^{\{h\} \times \Omega}=0, \\
\left|\left\{d \in D: x_{(h,(d, h))}^{*}=1\right\}\right| & =\bar{\delta}_{h}, \\
|\mu(h)| & =\bar{\delta}_{h} .
\end{aligned}
$$

We can deal with the case of $t_{h}^{*}>0$ in a paralell manner by using (31).

\subsubsection{Remark on the proof of Theorem 2}

Referees of the journal raised a concern about the length of the proof of Theorem 2, especially that of the implication (i) $\Rightarrow$ (ii), and proposed an alternative short proof. ${ }^{25}$ Here we explain their ideas, as well as the advantage of our proof.

A referee pointed out that the proof can be shortened by using the "L-convex intersection theorem," which states the optimality condition of the sum of two $\mathrm{L}^{\natural}$-convex functions. ${ }^{26}$ Consider two functions $g_{1}$ and $g_{2}$ defined over salaries $s$ and transfers $t$ such that

$$
g_{1}(s, t)=\sum_{d \in D} V_{d}(s)+G(t), g_{2}(s, t)=\sum_{h \in H} V_{h}(s, t) .
$$

Then, $F(s, t)=g_{1}(s, t)+g_{2}(s, t)$. Applying the L-convex intersection theorem to the two functions, we can prove the existence of a pair of salary/transfer systems satisfying the conditions stated in (ii). ${ }^{27}$

We fully admit that the referee's proof is shorter and easier to understand. Meanwhile, our proof appears to be more informative about how to construct

25 The author deeply appreciates the two referees for sending an alternative proof.

${ }^{26}$ The L-convex intersection theorem is a cousin of the M-convex intersection theorem (see Theorem 8.17 of Murota 2003a), replacing $\mathrm{M}^{\natural}$-convex functions in the latter theorem with $\mathrm{L}^{\natural}$-convex functions. This theorem is an immediate corollary of the L-separation theorem (see Theorem 8.16 of Murota 2003a).

${ }^{27}$ Another referee suggested adopting the techniques in Ausubel (2006) and Sun and Yang (2009). In their proofs, given a minimizer $p^{*}$ of the Lyapunov function, any efficient allocation is supported by $p^{*}$ as an equilibrium (a related claim is formerly established in Lemma 6 of Gul and Stacchetti (1999)). A parallel claim holds in our model: given a minimizer $\left(s^{*}, t^{*}\right)$, any constrained efficient matching is supported by $\left(s^{*}, t^{*}\right)$ as an equilibrium (this claim can be verified from the choice of $x^{*}$ after Claim 11 in the proof). It might be possible to prove this claim without relying on the saddle-point approach. 
the function $L(\cdot)$ (and the corresponding algorithm). As will be detailed in the proof, $L(\cdot)$ is derived by setting constraints in a visually simple way (see Fig. 3 in Section 5.3.2) and applying the discrete saddle-point theorem. Providing a recipe seems to be important in extending our approach to other socio-economic problems, such as those mentioned in Section 1.

\subsection{Proof of Theorem 3}

Let $d \in D$ and $h \in H$ be arbitrarily chosen. As the sum of $L^{\natural}$-convex functions is $L^{\natural}$-convex (see Theorem 7.11 of Murota 2003a), it suffices to prove the following: ${ }^{28}$

$$
\begin{aligned}
& V_{d}: \mathbb{Z}_{+}^{\Omega} \rightarrow \mathbb{Z} \text { is } \mathrm{L}^{\natural} \text {-convex. } \\
& V_{h}: \mathbb{Z}_{+}^{\Omega} \times \mathbb{Z}^{H} \rightarrow \mathbb{Z} \text { is } \mathrm{L}^{\natural} \text {-convex. } \\
& G_{h}: \mathbb{Z}^{H} \rightarrow \mathbb{Z} \text { is } \mathrm{L}^{\natural} \text {-convex. }
\end{aligned}
$$

To prove (49) and (50), it suffices to prove that $V_{d}: \mathbb{Z}^{\Omega} \rightarrow \mathbb{Z}$ and $V_{h}$ : $\mathbb{Z}^{\Omega} \times \mathbb{Z}^{H} \rightarrow \mathbb{Z}$ are $\mathrm{L}^{\natural}$-convex, because restricting the domain to non-negative vectors preserves $L^{\natural}$-convexity (see Theorem 7.11 of Murota (2003a)).

Proof of (49): We define $\hat{v}_{d}: \mathbb{Z}^{\Omega} \rightarrow \mathbb{Z} \cup\{-\infty\}$ by $\hat{v}_{d}(x)=\tilde{v}_{d}[\mathbf{0}](x)$ for all

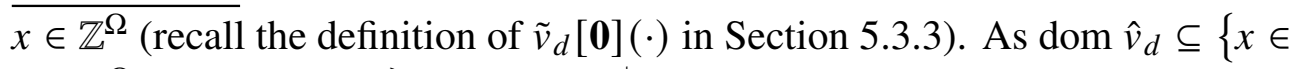
$\left.\{0,1\}^{\Omega}: \sum_{\omega \in \Omega} x_{\omega} \leq 1\right\},-\hat{v}_{d}(\cdot)$ is $\mathrm{M}^{\natural}$-convex. As $V_{d}(\cdot)$ is a convex conjugate function of $-\hat{v}_{d}(\cdot)$, by the discrete conjugacy theorem (see Theorem 8.12 of Murota (2003a)), $V_{d}(\cdot)$ is $\mathrm{L}^{\natural}$-convex.

Proof of (50): We define $\hat{v}_{h}: \mathbb{Z}^{\Omega} \rightarrow \mathbb{Z} \cup\{-\infty\}$ by $\hat{v}_{h}(x)=\tilde{v}_{h}[\mathbf{0 , 0}](x)$ for all $x \in \mathbb{Z}^{\Omega}$ (recall the definition of $\tilde{v}_{h}[\mathbf{0}, \mathbf{0}](\cdot)$ in Section 5.3.3). We define $\bar{v}_{h}: \mathbb{Z}^{\Omega} \times \mathbb{Z} \rightarrow \mathbb{Z} \cup\{-\infty\}$ by

$$
\bar{v}_{h}(x, r)= \begin{cases}\hat{v}_{h}(x) & \text { if } r=-\sum_{\omega \in \Omega} x_{\omega}, \\ -\infty & \text { otherwise. }\end{cases}
$$

Since $\hat{v}_{h}(\cdot)$ is $\mathbf{M}^{\natural}$-concave, $\bar{v}_{h}(\cdot, \cdot)$ satisfies a discrete concavity called $M$ concavity (see (6.4) of Murota (2003a)). Its concave conjugate function $\bar{v}_{h}^{0}(\cdot, \cdot)$

${ }^{28}$ As the domains of $V_{d}(\cdot)$ and $V_{h}(\cdot)$ are different, we cannot take their sum directly. However, this problem can be circumvented by extending the domain of $V_{d}(\cdot)$ to $\tilde{V}_{d}(\cdot, \cdot)$ defined by $\tilde{V}_{d}(s, t)=V_{d}(s)$ for all $(s, t) \in \mathbb{Z}_{+}^{\Omega} \times \mathbb{Z}^{H}$. One easily verifies that L ${ }^{\natural}$-convexity of $V_{d}(\cdot)$ is preserved in $\tilde{V}_{d}(\cdot, \cdot)$. Similarly, we can extend the domain of $G_{h}(\cdot)$. 
is given as follows: for any $\left(s, t_{h}\right) \in \mathbb{Z}^{\Omega} \times \mathbb{Z}$,

$$
\begin{aligned}
\bar{v}_{h}^{0}\left(s, t_{h}\right) & =-\sup _{(x, r) \in \mathbb{Z}^{\Omega} \times \mathbb{Z}}\left\{\bar{v}_{h}(x, r)-\left(s, t_{h}\right) \cdot(x, r)\right\} \\
& =-\sup _{x \in \mathbb{Z}^{\Omega}}\left\{\hat{v}_{h}(x)-s \cdot x-t_{h} \cdot\left(-\sum_{\omega \in \Omega} x_{\omega}\right)\right\} \\
& =-\sup _{x \in \mathbb{Z}^{\Omega}}\left\{\hat{v}_{h}(x)-s \cdot x+t_{h} \cdot \sum_{\omega \in \Omega} x_{\omega}\right\} \\
& =-V_{h}(s, t) .
\end{aligned}
$$

By the discrete conjugacy theorem (Theorem 8.12 of Murota (2003a)), $V_{h}(\cdot, \cdot)$ is L-convex. As L-convexity is stronger than $\mathrm{L}^{\natural}$-convexity, $V_{h}(\cdot, \cdot)$ is $\mathrm{L}^{\natural}$-convex. Proof of (51): As described in Fig. $1, G_{h}(\cdot)$ satisfies

$$
G_{h}\left(t_{h}-1\right)+G_{h}\left(t_{h}+1\right) \geq 2 G_{h}\left(t_{h}\right) \text { for all } t_{h} \in \mathbb{Z},
$$

which is equivalent to $\mathrm{L}^{\natural}$-convexity (and also to $\mathrm{M}^{\natural}$-convexity) in the 1 dimensional case; see Section 1.2 of Murota (2003a) for a detailed discussion.

\section{References}

Ausubel, L.M., 2006. An efficient dynamic auction for heterogeneous commodities. American Economic Review 96, 602-629.

Baumol, W.J., Oates, W.E., 1971. The use of standards and prices for protection of the environment. Swedish Journal of Economics 73, 42-54.

Burch, I., Gilchrist, J., 2018. Survey of global activity to phase out internal combustion engine vehicles. Center of Climate Protection: Santa Rosa, CA, USA .

Candogan, O., Epitropou, M., Vohra, R.V., 2016. Competitive equilibrium and trading networks: A network flow approach, in: Proceedings of the 2016 ACM Conference on Economics and Computation, ACM. pp. 701-702.

Dupuy, A., Galichon, A., Jaffe, S., Kominers, S.D., 2020. Taxation in matching markets. International Economic Review, forthcoming .

Fujishige, S., Tamura, A., 2007. A two-sided discrete-concave market with possibly bounded side payments: An approach by discrete convex analysis. Mathematics of Operations Research 32, 136-155.

Fujishige, S., Yang, Z., 2003. A note on Kelso and Crawford's gross substitutes condition. Mathematics of Operations Research 28(3), 463-469. 
Gul, F., Stacchetti, E., 1999. Walrasian equilibrium with gross substitutes. Journal of Economic Theory 87(1), 95-124.

Hatfield, J.W., Kominers, S., Nichifor, A., Ostrovsky, M., Westkamp, A., 2013. Stability and competitive equilibrium in trading networks. Journal of Political Economy 121(5), 966-1005.

Hatfield, J.W., Kominers, S.D., Nichifor, A., Ostrovsky, M., Westkamp, A., 2019. Full substitutability. Theoretical Economics 14, 1535-1590.

Hiriart-Urruty, J.B., Lemaréchal, C., 2001. Fundamentals of convex analysis. Springer. Ikebe, Y.T., Tamura, A., 2015. Stability in supply chain networks: An approach by discrete convex analysis. Journal of the Operations Research Society of Japan 58, 271-290.

Kamada, Y., Kojima, F., 2017. Recent developments in matching with constraints. American Economic Review 107, 200-204.

Kamada, Y., Kojima, F., 2020. Accommodating various policy goals in matching with constraints. Japanese Economic Review 71, 101-133.

Kelso, A., Crawford, V., 1982. Job matching, coalition formation, and gross substitutes. Econometrica , 1483-1504.

Kojima, F., Sun, N., Yu, N.N., 2019. Job matching under constraints. Working Paper (version: July 29, 2019) .

Kojima, F., Sun, N., Yu, N.N., 2020a. Job matching under constraints. American Economic Review, forthcoming .

Kojima, F., Sun, N., Yu, N.N., 2020b. Job matching with subsidy and taxation. Working Paper .

Kojima, F., Tamura, A., Yokoo, M., 2018. Designing matching mechanisms under constraints: An approach from discrete convex analysis. Journal of Economic Theory 176, 803-833.

Kolmogorov, V., Shioura, A., 2009. New algorithms for convex cost tension problem with application to computer vision. Discrete Optimization 6, 378-393.

Murota, K., 1998. Discrete convex analysis. Mathematical Programming 83, 313-371.

Murota, K., 2003a. Discrete convex analysis. Society for Industrial and Applied Mathematics.

Murota, K., 2003b. On steepest descent algorithms for discrete convex functions. SIAM Journal on Optimization 14, 699-707.

Murota, K., 2016. Discrete convex analysis: A tool for economics and game theory. Journal of Mechanism and Institution Design 1, 151-273.

Murota, K., Shioura, A., 2014. Exact bounds for steepest descent algorithms of 1-convex function minimization. Operations Research Letters 42, 361-366.

Murota, K., Shioura, A., Yang, Z., 2016. Time bounds for iterative auctions: a unified approach by discrete convex analysis. Discrete Optimization 19, 36-62. 
Parry, I.W., Walls, M., Harrington, W., 2007. Automobile externalities and policies. Journal of Economic Literature 45, 373-399.

Rockafellar, R.T., 1970. Convex analysis. Princeton University Press.

Sun, N., Yang, Z., 2009. A double-track adjustment process for discrete markets with substitutes and complements. Econometrica 77, 933-952.

Tiel, J.v., 1984. Convex analysis: An introductory text. John Wiley.

Yokote, K., 2020. Supplemental material for "On optimal taxes and subsidies: A discrete saddle-point theorem with application to job matching under constraints". Available at https://sites.google.com/site/kojiyokote/. 